\title{
Geochemical study of groundwater in the Sho river fan, Toyama Prefecture for heat usage by geothermal heat pump
}

\author{
Kaname Iwatake, ${ }^{1} *$ Toshiaki Mizoguchi, ${ }^{2}$ Shingo Tomiyama,${ }^{3}$ Jing Zhang, ${ }^{1}$ \\ HiRoshi SATAKE ${ }^{1 \dagger}$ and AKIRA UEDA ${ }^{1}$ \\ ${ }^{1}$ Department of Environmental Biology and Chemistry, University of Toyama, Gofuku 3190, Toyama 930-8555, Japan \\ ${ }^{2}$ Toyama Prefectural Environment Science Research Center, 17-1 Taikoyama, Imizu, Toyama 939-0363, Japan \\ ${ }^{3}$ Mitsubishi Materials Techno Corporation, 1-297 Kitabukuro-cho, Omiya-ku, Saitama 330-0835, Japan
}

(Received July 4, 2012; Accepted June 8, 2013)

\begin{abstract}
Chemical and isotopic $\left(\mathrm{D},{ }^{18} \mathrm{O}\right)$ compositions of 40 shallow groundwater, 14 observation well water and 2 river water samples in the Sho river fan, Toyama, northern part of central Japan, were analyzed to examine their water quality, origins and water flow in order to obtain baseline geochemical data for application of groundwater to geothermal heat pump (Geo-HP). Water temperatures of the groundwaters in the observation wells are almost constant at $15^{\circ} \mathrm{C}$ from surface to $c a .60 \mathrm{~m}$ in depth and increase up to $25^{\circ} \mathrm{C}$ at depth of $200 \mathrm{~m}$ with the thermal gradient of $6^{\circ} \mathrm{C} / 100 \mathrm{~m}$. This geothermal gradient is twice as high as that of the other areas in Japan, suggesting existence of high temperature geothermal sources beneath this region and a high potential for heat usage in winter.

The isotopic results show that the groundwaters are a mixture of two big river waters (Sho and Oyabe rivers) and precipitation in the Sho river fan and that the contribution of infiltration from the Sho river has increased in recent 10 years. The Sho river has lower $\delta^{18} \mathrm{O}$ values than the Oyabe river. The groundwaters shallower than $80 \mathrm{~m} \mathrm{depth}$ are of a Ca$\mathrm{HCO}_{3}$ type. Deep groundwaters from observation wells were characterized by high in $\mathrm{pH}$ and $\mathrm{HCO}_{3}$ compared to the shallow groundwaters due to ion exchange with rocks containing clay minerals. The deep groundwaters are almost saturated or oversaturated with respect to calcite and dolomite, becoming confined due to the presence of an impermeable layer. In this paper, formation of calcite and iron hydroxide scale inside pipes of the Geo-HP was discussed from the chemical compositions of groundwater to examine whether the water is applicable to geothermal heat pump or not.
\end{abstract}

Keywords: groundwater, heat usage, hydrogen isotope, oxygen isotope, scale, geothermal heat pump

\section{INTRODUCTION}

The minimum and maximum air temperature is $0^{\circ} \mathrm{C}$ in winter and $35^{\circ} \mathrm{C}$ in summer in central areas along the Japan Sea such as Toyama Prefecture, northern Central Japan. Also, a large amount of snow falls in winter. Most residents in Toyama use kerosene and electricity for house-heating and pay more than 500 USD per year for kerosene. Since the gigantic Tohoku earthquake of March 11, 2011 that resulted in the loss of the Fukushima Daiichi Nuclear power station, saving electric power has become an urgent necessity in Japan and alternative energy resources are being sought. In Toyama Prefecture, a large amount ( 1 million tons/year) of groundwater flows from the mountains ( $c a .3000 \mathrm{~m}$ in altitude) to the Sea of Japan side within $100 \mathrm{~km}$ in distance (Toyama Prefecture,

\footnotetext{
*Corresponding author (e-mail: m1241504@ems.u-toyama.ac.jp)

${ }^{\dagger}$ Deceased November 2009.
}

Copyright (c) 2013 by The Geochemical Society of Japan.
2006), thus most rivers in Toyama Prefecture have the highest hydraulic gradient in Japan. Since the water temperature of shallow groundwater in Toyama is constant at $15^{\circ} \mathrm{C}$ throughout the year, the groundwater can be used for heat source of air conditioning of houses and industry in winter by using the temperature difference between air and groundwater.

The use of the geothermal heat pump (Geo-HP) is now spreading worldwide (e.g., Huttrer, 1997; Diao et al., 2004; Lee and Hahn, 2006; Fujii et al., 2007; Russo et al., 2011). In particular, the open-type Geo-HP using groundwater has been often used because of the low cost (e.g., Wang et al., 2012). In the open-type Geo-HP system, groundwater is pumped to the surface, heatexchanged and returned to the aquifer through a reinjection well. In the closed-type Geo-HP system, antifreezing fluid circulates through the U-tube installed in the underground to pick up the heat. The final goal of our study is to evaluate the applicability of groundwater as a heat source to air conditioning of houses and melting of road snow in this area. In summer, shallow groundwater, which is cooler than the atmospheric temperature, can be 


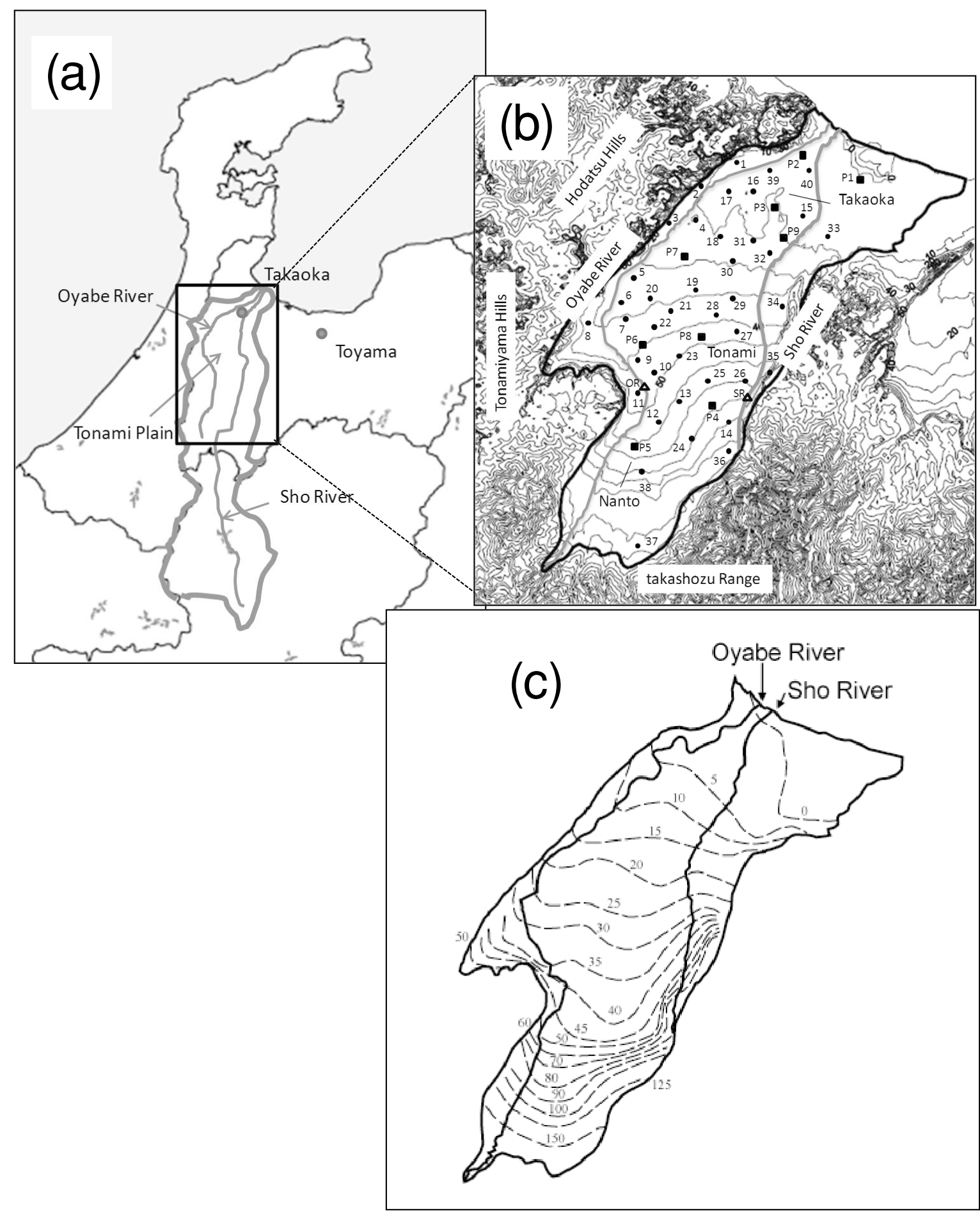

Fig. 1. Locality of the Sho river fan. (a) Watershed of the Sho river fan. (b) The detailed study area and sample localities of shallow groundwaters and river water in the Sho river fan. Solid circles indicate the shallow groundwater, solid squares the observation well, open triangles river water. Numbers in the sample locality correspond to those in Tables 1, 2 and 3. (c) Groundwater levels in the Sho river fan (modified from the Toyama Prefecture, 2006). 
used for cooling houses. In this system, the quality and mode of water movement are important parameters for the use of groundwater. In addition, the effect of temperature change of groundwater after heat exchange on the later available heat should also be examined (e.g., Freedman et al., 2012; Ni et al., 2011). A constant supply of groundwater is required for the long use of the GeoHP device. The quality of groundwater is also important to keep high heat exchange efficiency during the operation. Precipitation of some chemical components such as iron and calcium carbonate as a scale in the heat exchanger and water pipes has to be avoided (e.g., Andritsos and Karabelas, 2003; Lei et al., 2011). Iron in anoxic groundwater is dissolved as ferrous ion $\left(\mathrm{Fe}^{2+}\right)$ and oxidized to ferric ion $\left(\mathrm{Fe}^{3+}\right)$ by oxidation upon contact with atmosphere and precipitates as iron hydroxide $\left(\mathrm{Fe}(\mathrm{OH})_{3}\right)$ and iron carbonate $\left(\mathrm{FeCO}_{3}\right)$.

There are many works done on water movement in alluvium fan in the world (e.g., Mizutani and Oda, 1983; Li et al., 2008; Hidalgo and Cruz-Sanjulian, 2001; Mizutani et al., 2001; Chkirbene et al., 2009; Yuan et al., 2011; Vanderzalm et al., 2011; Tsuchihara et al., 2011). In this study, groundwaters and river waters in the Sho river fan were geochemically analyzed to determine the water flow and quality of groundwater in order to assess the potential use of the groundwater for Geo-HP. The statistics data such as water level fluctuation, flux and movement of groundwater in the Sho River fan should be consistent with the geochemical data (e.g., Tomiyama et al., $2009,2010,2011)$. In the Sho river fan, groundwater has been used for industry, snow melting on the road and drinking. Excessive extraction of groundwater in the past resulted in the land subsidence in the northern part of the fan, creating permanent damage to the land surface. Such land subsidence has been reported elsewhere and the correlation between groundwater level and the change in morphology in the land subsidence has also been studied (e.g., Chen et al., 2010).

The stable isotopic compositions of groundwater and river waters in the Sho river fan have been determined previously (Mizutani and Oda, 1983; Kato et al., 1984; Takaoka and Mizutani, 1987; Mizutani et al., 1987; Miyamoto, 2000). Mizutani and Oda (1983) reported that a significant difference exists in $\delta \mathrm{D}$ and $\delta^{18} \mathrm{O}$ values between the waters of the Sho and Oyabe rivers and indicated that the aquifers in the eastern half of the Sho river fan were recharged by the Sho river water and those in the western half by the Oyabe river water. They also noted contribution of the precipitation and finally demonstrated 5 water sources for groundwaters in the Sho river fan.

\section{Sample locality and Analytical Procedures}

The Sho river fan is located in the western part of
Toyama prefecture and spreads between Nanto and Takaoka cities (Fig. 1a), where two big rivers, Sho and Oyabe, flow along the edge of the fan (Fig. 1b). The geology of the study area consists of unconsolidated coarsegrained andesitic breccia derived from the surrounding mountains. Confined aquifer does not exist between Nanto and Takaoka, because there is no permeable clay layers in the gravel layer. The permeability of the gravel layer is reported as $10^{-2} \mathrm{~cm} / \mathrm{sec}$ (Toyama Prefecture (2006). Two or three clay layers are present between the two rivers in the northern, sea side part of the fan, where the lower aquifer is confined. In some areas of Takaoka city, water head is $c a .2 \mathrm{~m}$ above the ground surface. The Sho and Oyabe rivers originate from mountains with the altitude of $1,625 \mathrm{~m}$ and $1,572 \mathrm{~m}$ and flow to the ocean $115 \mathrm{~km}$ and $68 \mathrm{~km}$ away with the flow rates of $34 \mathrm{~m}^{3} / \mathrm{sec}$ and $24.3 \mathrm{~m}^{3} / \mathrm{sec}$, respectively (Fig. 1a). The highest elevation of the catchment area is $1,020 \mathrm{~m}$ for the Sho river and $260 \mathrm{~m}$ for the Oyabe river. Annual precipitation is 3,200 $\mathrm{mm}$ at Shirakawa where the Sho river originates, and 2,300 $\mathrm{mm}$ in Takaoka area (ocean side). The size of the Sho and Oyabe rivers area are $1,180 \mathrm{~km}^{2}$ and $682 \mathrm{~km}^{2}$, respectively. The groundwater levels are shown in Fig. 1c which indicates groundwaters flowing radially from top to end of the fan. The flow velocity of the shallow groundwater (above $60 \mathrm{~m}$ depth) has been estimated to be $250 \mathrm{~m} /$ day at Tonami, the top area of the fan along Sho river (Takahashi et al., 2003). They used deuteriumenriched and $\mathrm{NaCl}$ solutions to evaluate the velocity. Mizutani and Oda (1983) estimated the water flow rate of 10 to $13 \mathrm{~m} /$ day for the deep groundwater (confined aquifer) in the northern part of the fan.

The study area and water sampling sites are provided in Fig. 1b. Three types of water samples are collected; shallow groundwater, groundwater from observation wells, and river waters. In this study, the groundwaters outside the fan (the vicinity of Takaoka) were also investigated, for the area around Takaoka was not included in the previous study. They were analyzed for their chemical and isotopic compositions. Water samples were collected in $100 \mathrm{ml}$ and $250 \mathrm{ml}$ plastic bottles. Water temperature, $\mathrm{pH}, \mathrm{EC}$, and Eh were measured at the sampling site using a thermometer, a pH meter (Shindengen, KS701), EC meter (Horiba, B-173) and Eh meter (TOA, RM12P), respectively. Alkalinity was determined using standard titration with $\mathrm{HCl}$. Dissolved ion concentrations were measured using conventional methods. The major cations $(\mathrm{Na}, \mathrm{K}, \mathrm{Ca}, \mathrm{Mg})$ and anions $\left(\mathrm{Cl}, \mathrm{NO}_{3}, \mathrm{SO}_{4}\right)$ in the filtered samples were analyzed with an ion chromatograph (Metrohm 761 Compact IC). The charge balances of the obtained results are within $\pm 5 \%$ and the analytical uncertainties on dissolved ion concentrations are also within $5 \%$. $\mathrm{SiO}_{2}$ was analyzed with a UV-VIS spectrophotometer (SHIMADZU UVmini-1240). Iron concentration was 
Table 1. Sample locality, temperature, $p H, E C$ and ORP of shallow groundwaters and river waters in the Sho River fan area

\begin{tabular}{|c|c|c|c|c|c|c|c|c|}
\hline Sample name & Sample date & Latitude & Longitude & Altitude & Temp. $\left({ }^{\circ} \mathrm{C}\right)$ & $\mathrm{pH}$ & $\mathrm{EC}(\mathrm{mS} / \mathrm{m})$ & ORP $(\mathrm{mV}) \mathrm{Pt}^{*}$ \\
\hline 1 & 2011.8 .12 & $36^{\circ} 45^{\prime} 19.10^{\prime \prime}$ & $136^{\circ} 58^{\prime} 19.44^{\prime \prime}$ & 7 & 21.3 & 7.62 & 29.5 & -9 \\
\hline 2 & 2011.8 .12 & $36^{\circ} 44^{\prime} 16.81^{\prime \prime}$ & $136^{\circ} 56^{\prime} 41.87^{\prime \prime}$ & 11 & 14.5 & 7.46 & 37.1 & 79 \\
\hline 3 & 2011.8 .12 & $36^{\circ} 43^{\prime} 0.54^{\prime \prime}$ & $136^{\circ} 55^{\prime} 13.97^{\prime \prime}$ & 16 & 25.0 & 7.50 & 10.0 & 81 \\
\hline 4 & 2011.8 .12 & $36^{\circ} 43^{\prime} 9.70^{\prime \prime}$ & $136^{\circ} 56^{\prime} 34.29^{\prime \prime}$ & 12 & 15.6 & 7.77 & 25.3 & 86 \\
\hline 5 & 2011.8.12 & $36^{\circ} 41^{\prime} 43.97^{\prime \prime}$ & $136^{\circ} 54^{\prime} 12.96^{\prime \prime}$ & 21 & 15.8 & 7.09 & 19.6 & 109 \\
\hline 6 & 2011.8 .12 & $36^{\circ} 40^{\prime} 45.53^{\prime \prime}$ & $136^{\circ} 53^{\prime} 9.19^{\prime \prime}$ & 26 & 18.0 & 6.88 & 18.7 & 139 \\
\hline 7 & 2011.8 .12 & $36^{\circ} 39^{\prime} 39.21^{\prime \prime}$ & $136^{\circ} 53^{\prime} 34.68^{\prime \prime}$ & 31 & 16.4 & 6.20 & 14.8 & 138 \\
\hline 8 & 2011.8.12 & $36^{\circ} 39^{\prime} 15.22^{\prime \prime}$ & $136^{\circ} 51^{\prime} 26.47^{\prime \prime}$ & 34 & 13.6 & 7.57 & 13.0 & 104 \\
\hline 9 & 2011.8.12 & $36^{\circ} 37^{\prime} 49.74^{\prime \prime}$ & $136^{\circ} 53^{\prime} 35.45^{\prime \prime}$ & 40 & 17.5 & 7.61 & 11.1 & 69 \\
\hline 10 & 2011.8 .12 & $36^{\circ} 37^{\prime} 25.66^{\prime \prime}$ & $136^{\circ} 54^{\prime} 56.93^{\prime \prime}$ & 47 & 17.1 & 7.58 & 12.2 & 93 \\
\hline 11 & 2011.8 .12 & $36^{\circ} 36^{\prime} 51.76^{\prime \prime}$ & $136^{\circ} 53^{\prime} 51.05^{\prime \prime}$ & 45 & 14.0 & 6.93 & 16.4 & 85 \\
\hline 12 & 2011.8.12 & $36^{\circ} 35^{\prime} 51.96^{\prime \prime}$ & $136^{\circ} 55^{\prime} 4.90^{\prime \prime}$ & 55 & 16.0 & 6.36 & 14.5 & 93 \\
\hline 13 & 2011.8 .12 & $36^{\circ} 36^{\prime} 35.32^{\prime \prime}$ & $136^{\circ} 56^{\prime} 32.81^{\prime \prime}$ & 64 & 21.4 & 7.08 & 16.1 & 119 \\
\hline 14 & 2011.8 .12 & $36^{\circ} 35^{\prime} 59.01^{\prime \prime}$ & $136^{\circ} 59^{\prime} 7.19^{\prime \prime}$ & 83 & 16.1 & 7.60 & 13.8 & 103 \\
\hline 15 & 2011.8 .27 & $36^{\circ} 43^{\prime} 57.93^{\prime \prime}$ & $137^{\circ} 1^{\prime} 39.54^{\prime \prime}$ & 11 & 15.4 & 7.89 & 11.7 & 75 \\
\hline 16 & 2011.8.27 & $36^{\circ} 44^{\prime} 11.93^{\prime \prime}$ & $136^{\circ} 59^{\prime} 53.02^{\prime \prime}$ & 7 & 14.0 & 7.57 & 12.0 & 67 \\
\hline 17 & 2011.8 .27 & $36^{\circ} 44^{\prime} 0.96^{\prime \prime}$ & $136^{\circ} 58^{\prime} 25.27^{\prime \prime}$ & 8 & 14.2 & 7.60 & 17.7 & 86 \\
\hline 18 & 2011.8 .27 & $36^{\circ} 42^{\prime} 42.85^{\prime \prime}$ & $136^{\circ} 57^{\prime} 56.37^{\prime \prime}$ & 14 & 14.0 & 7.56 & 16.8 & 108 \\
\hline 19 & 2011.8 .27 & $36^{\circ} 41^{\prime} 0.33^{\prime \prime}$ & $136^{\circ} 56^{\prime} 43.72^{\prime \prime}$ & 26 & 15.9 & 6.58 & 18.1 & 126 \\
\hline 20 & 2011.8 .27 & $36^{\circ} 40^{\prime} 53.73^{\prime \prime}$ & $136^{\circ} 54^{\prime} 52.16^{\prime \prime}$ & 25 & 14.8 & 6.73 & 18.9 & 146 \\
\hline 21 & 2011.8 .27 & $36^{\circ} 40^{\prime} 12.83^{\prime \prime}$ & $136^{\circ} 55^{\prime} 27.11^{\prime \prime}$ & 31 & 17.3 & 7.72 & 16.7 & 95 \\
\hline 22 & 2011.8 .27 & $36^{\circ} 39^{\prime} 21.81^{\prime \prime}$ & $136^{\circ} 54^{\prime} 44.75^{\prime \prime}$ & 38 & 14.4 & 6.67 & 13.7 & 100 \\
\hline 23 & 2011.8 .27 & $36^{\circ} 38^{\prime} 1.36^{\prime \prime}$ & $136^{\circ} 56^{\prime} 8.83^{\prime \prime}$ & 50 & 16.3 & 6.55 & 17.9 & 68 \\
\hline 24 & 2011.8 .27 & $36^{\circ} 35^{\prime} 2.99^{\prime \prime}$ & $136^{\circ} 56^{\prime} 44.91^{\prime \prime}$ & 78 & 13.7 & 6.67 & 11.1 & 127 \\
\hline 25 & 2011.8 .27 & $36^{\circ} 37^{\prime} 28.79^{\prime \prime}$ & $136^{\circ} 57^{\prime} 39.45^{\prime \prime}$ & 61 & 26.3 & 6.55 & 15.7 & 120 \\
\hline 26 & 2011.8 .27 & $36^{\circ} 37^{\prime} 55.59^{\prime \prime}$ & $136^{\circ} 59^{\prime} 18.91^{\prime \prime}$ & 59 & 16.1 & 7.33 & 9.7 & 120 \\
\hline 27 & 2011.8 .27 & $36^{\circ} 39^{\prime} 26.96^{\prime \prime}$ & $136^{\circ} 58^{\prime} 49.95^{\prime \prime}$ & 42 & 18.6 & 6.58 & 13.4 & 112 \\
\hline 28 & 2011.8 .27 & $36^{\circ} 39^{\prime} 46.10^{\prime \prime}$ & $136^{\circ} 57^{\prime} 32.66^{\prime \prime}$ & 37 & 14.9 & 6.99 & 16.1 & 116 \\
\hline 29 & 2011.8 .27 & $36^{\circ} 40^{\prime} 27.09^{\prime \prime}$ & $136^{\circ} 58^{\prime} 24.55^{\prime \prime}$ & 30 & 15.8 & 6.74 & 16.0 & 720 \\
\hline 30 & 2011.8 .27 & $36^{\circ} 41^{\prime} 43.58^{\prime \prime}$ & $136^{\circ} 58^{\prime} 29.55^{\prime \prime}$ & 21 & 15.2 & 6.56 & 12.7 & 374 \\
\hline 31 & 2011.8 .27 & $36^{\circ} 42^{\prime} 41.37^{\prime \prime}$ & $136^{\circ} 59^{\prime} 26.01^{\prime \prime}$ & 15 & 13.9 & 7.15 & 26.0 & 315 \\
\hline 32 & 2011.8.27 & $36^{\circ} 42^{\prime} 4.72^{\prime \prime}$ & $137^{\circ} 0^{\prime} 7.12^{\prime \prime}$ & 17 & 12.2 & 7.92 & 8.9 & 286 \\
\hline 33 & 2011.9 .7 & $36^{\circ} 43^{\prime} 17.08^{\prime \prime}$ & $137^{\circ} 3^{\prime} 15.12^{\prime \prime}$ & 10 & 14.9 & 6.48 & 13.7 & 217 \\
\hline 34 & 2011.9 .7 & $36^{\circ} 40^{\prime} 4.13^{\prime \prime}$ & $137^{\circ} 1^{\prime} 31.81^{\prime \prime}$ & 36 & 14.4 & 6.77 & 10.5 & 183 \\
\hline 35 & 2011.9.7 & $36^{\circ} 37^{\prime} 15.54^{\prime \prime}$ & $137^{\circ} 0^{\prime} 36.40^{\prime \prime}$ & 70 & 21 & 6.26 & 14.1 & 163 \\
\hline 36 & 2011.9.7 & $36^{\circ} 34^{\prime} 15.01^{\prime \prime}$ & $136^{\circ} 59^{\prime} 5.85^{\prime \prime}$ & 114 & 18.2 & 6.7 & 8.9 & 186 \\
\hline 37 & 2011.9 .7 & $36^{\circ} 30^{\prime} 20.74^{\prime \prime}$ & $136^{\circ} 54^{\prime} 8.11^{\prime \prime}$ & 169 & 14.1 & 7.4 & 28.7 & 37 \\
\hline 38 & 2011.9 .7 & $36^{\circ} 33^{\prime} 22.68^{\prime \prime}$ & $136^{\circ} 54^{\prime} 19.31^{\prime \prime}$ & 81 & 14.8 & 6.52 & 10.5 & 201 \\
\hline 39 & 2011.9.7 & $36^{\circ} 45^{\prime} 36.03^{\prime \prime}$ & $137^{\circ} 0^{\prime} 24.23^{\prime \prime}$ & 4 & 17.3 & 6.57 & 88.0 & 9 \\
\hline 40 & 2011.9.7 & $36^{\circ} 45^{\prime} 28.29^{\prime \prime}$ & $137^{\circ} 2^{\prime} 54.69^{\prime \prime}$ & 3 & 15.4 & 7.48 & 13.3 & 114 \\
\hline SR & 2011.8 .12 & $36^{\circ} 37^{\prime} 20.84^{\prime \prime}$ & $136^{\circ} 59^{\prime} 57.74^{\prime \prime}$ & 63 & 24.9 & 7.69 & 8.2 & 102 \\
\hline OR & 2011.8 .12 & $36^{\circ} 37^{\prime} 3.52^{\prime \prime}$ & $136^{\circ} 53^{\prime} 56.18^{\prime \prime}$ & 45 & 23.5 & 7.79 & 13.7 & 61 \\
\hline
\end{tabular}

* Measured with a Pt electrode.

determined by ICP-MS at the Naka Institute of Mitsubishi Materials Corp.

The hydrogen and oxygen isotope ratios were determined by a stable isotope analyzer for $\mathrm{H}_{2} \mathrm{O}$ by the use of a diode laser-based infrared spectrometer. The results were reported relative to $\mathrm{V}-\mathrm{SMOW} 2$ with an analytical precision of $1 \%$ for $\delta \mathrm{D}$ and $0.3 \%$ for $\delta^{18} \mathrm{O}$, respectively. 
Table 2. Chemical and stable isotope compositions of shallow groundwaters and river waters in Sho River fan area

\begin{tabular}{|c|c|c|c|c|c|c|c|c|c|c|c|c|}
\hline \multirow[t]{2}{*}{ Sample name } & $\mathrm{Na}^{+}$ & $\mathrm{K}^{+}$ & $\mathrm{Mg}^{2+}$ & $\mathrm{Ca}^{2+}$ & $\mathrm{Cl}^{-}$ & $\mathrm{NO}_{3}^{-}$ & $\mathrm{SO}_{4}{ }^{2-}$ & $\mathrm{HCO}_{3}^{-}$ & $\mathrm{SiO}_{2}$ & \multirow{2}{*}{$\begin{array}{c}\mathrm{Fe} \\
\mu \mathrm{g} / \mathrm{L}\end{array}$} & $\delta \mathrm{D}$ & $\delta^{18} \mathrm{O}$ \\
\hline & \multicolumn{9}{|c|}{$\mathrm{mg} / \mathrm{L}$} & & \multicolumn{2}{|c|}{$\%$} \\
\hline 1 & 19.21 & 3.77 & 14.29 & 20.73 & 13.43 & 0.59 & 6.04 & 153.72 & 37.24 & 2.51 & -47 & -8.5 \\
\hline 2 & 12.37 & 2.13 & 6.23 & 62.97 & 13.42 & 0.86 & 4.16 & 215.94 & 31.51 & 2.38 & -45.8 & -8.1 \\
\hline 3 & 11.24 & 4.69 & 1.24 & 3.85 & 10.80 & 3.10 & 3.26 & 28.79 & 59.77 & 3.57 & -47.6 & -8.8 \\
\hline 4 & 8.98 & 1.34 & 5.88 & 37.00 & 8.82 & 7.74 & 7.95 & 129.32 & 13.39 & 2.38 & -55.2 & -9.2 \\
\hline 5 & 8.07 & 1.00 & 5.34 & 25.04 & 10.37 & 2.83 & 10.41 & 88.45 & 31.29 & 2.38 & -52.5 & -8.9 \\
\hline 6 & 9.11 & 0.99 & 6.12 & 22.71 & 8.63 & 2.63 & 10.08 & 87.84 & 29.54 & 2.38 & -53.6 & -9.1 \\
\hline 7 & 7.01 & 1.08 & 2.95 & 16.86 & 8.28 & 4.43 & 10.29 & 50.02 & 20.41 & 2.41 & -56.5 & -9.5 \\
\hline 8 & 7.72 & 7.15 & 4.72 & 4.92 & 11.14 & 24.41 & 12.39 & 9.76 & 16.68 & 2.40 & -46.5 & -8.7 \\
\hline 9 & 6.57 & 0.88 & 2.45 & 10.15 & 6.16 & 3.50 & 6.54 & 38.43 & 15.04 & 2.38 & -54.7 & -9.3 \\
\hline 10 & 7.44 & 0.88 & 2.77 & 12.53 & 8.44 & 3.93 & 7.69 & 42.09 & 19.15 & 2.38 & -53.6 & -9.2 \\
\hline 11 & 10.41 & 0.93 & 4.64 & 16.22 & 8.26 & 4.46 & 7.55 & 73.20 & 29.60 & 2.38 & -49.8 & -8.7 \\
\hline 12 & 8.68 & 0.78 & 4.42 & 13.65 & 7.26 & 3.72 & 7.36 & 59.17 & 17.54 & 2.40 & -53.8 & -8.9 \\
\hline 13 & 6.20 & 1.09 & 2.81 & 21.71 & 6.78 & 5.90 & 11.10 & 62.22 & 19.94 & 2.42 & -53.2 & -9.0 \\
\hline 14 & 4.96 & 0.85 & 2.73 & 19.61 & 5.30 & 3.02 & 7.48 & 62.22 & 14.66 & 2.39 & -61.2 & -10.4 \\
\hline 15 & 5.21 & 0.77 & 2.55 & 15.16 & 4.11 & 1.45 & 7.40 & 54.26 & 22.50 & 2.38 & -64 & -10.5 \\
\hline 16 & 6.09 & 0.81 & 2.72 & 15.08 & 4.02 & 1.76 & 6.42 & 55.87 & 20.41 & 2.41 & -62.6 & -10.4 \\
\hline 17 & 6.81 & 1.04 & 4.62 & 22.99 & 5.79 & 3.81 & 8.59 & 84.18 & 20.64 & 2.42 & -59.6 & -9.7 \\
\hline 18 & 6.48 & 1.11 & 3.66 & 21.22 & 7.32 & 5.69 & 10.68 & 68.59 & 20.89 & 2.37 & -56.2 & -9.0 \\
\hline 19 & 7.06 & 1.22 & 3.10 & 24.18 & 8.48 & 7.07 & 13.33 & 67.07 & 19.55 & 2.39 & -51.3 & -9.0 \\
\hline 20 & 8.45 & 1.32 & 3.79 & 22.97 & 16.17 & 4.80 & 13.19 & 59.78 & 23.93 & 2.42 & -50.8 & -9.0 \\
\hline 21 & 7.79 & 1.36 & 2.63 & 21.58 & 8.76 & 5.73 & 17.79 & 53.04 & 20.39 & 2.53 & -51.8 & -8.9 \\
\hline 22 & 7.22 & 0.95 & 2.01 & 15.28 & 9.39 & 3.88 & 11.72 & 43.04 & 17.98 & 2.38 & -53.4 & -9.0 \\
\hline 23 & 6.51 & 1.11 & 3.63 & 24.84 & 7.14 & 5.23 & 9.58 & 74.42 & 19.69 & 2.61 & -54.2 & -9.3 \\
\hline 24 & 4.88 & 1.36 & 1.70 & 13.31 & 4.96 & 7.44 & 8.50 & 33.55 & 10.60 & 2.37 & -55.7 & -9.2 \\
\hline 25 & 5.66 & 1.05 & 3.02 & 21.19 & 6.27 & 4.80 & 9.18 & 64.25 & 18.61 & 2.39 & -54.4 & -9.4 \\
\hline 26 & 4.13 & 0.78 & 1.76 & 11.71 & 3.89 & 2.02 & 8.21 & 36.36 & 12.65 & 2.39 & -62.3 & -10.0 \\
\hline 27 & 5.16 & 0.88 & 2.50 & 12.98 & 4.70 & 2.89 & 8.92 & 48.80 & 17.13 & 2.39 & -60.9 & -9.8 \\
\hline 28 & 5.76 & 1.02 & 3.30 & 21.31 & 6.51 & 4.41 & 10.06 & 64.66 & 18.17 & 2.40 & -57.4 & -9.7 \\
\hline 29 & 6.62 & 1.03 & 3.17 & 19.80 & 7.56 & 4.91 & 10.76 & 62.05 & 19.37 & 3.09 & -56.9 & -9.3 \\
\hline 30 & 5.63 & 1.18 & 1.71 & 15.92 & 6.69 & 5.91 & 10.07 & 34.16 & 14.88 & 2.39 & -56 & -9.7 \\
\hline 31 & 24.35 & 1.46 & 3.32 & 21.89 & 8.80 & 4.50 & 79.95 & 34.51 & 14.55 & 2.39 & -62 & -10.1 \\
\hline 32 & 5.09 & 0.61 & 1.64 & 12.36 & 3.72 & 1.42 & 9.66 & 40.87 & 21.59 & 2.38 & -64.6 & -10.6 \\
\hline 33 & 8.15 & 1.11 & 3.99 & 12.80 & 8.34 & 5.20 & 10.15 & 45.26 & 21.14 & 2.40 & -52 & -9.3 \\
\hline 34 & 5.58 & 0.94 & 2.06 & 11.38 & 5.78 & 2.43 & 7.77 & 37.75 & 13.29 & 2.39 & -58.5 & -9.9 \\
\hline 35 & 9.12 & 1.28 & 3.24 & 12.81 & 12.44 & 3.39 & 8.29 & 44.50 & 15.30 & 2.62 & -55 & -9.0 \\
\hline 36 & 5.11 & 1.35 & 1.92 & 7.98 & 7.31 & 3.04 & 6.41 & 24.68 & 10.18 & 2.39 & -62.1 & -9.8 \\
\hline 37 & 11.75 & 1.32 & 10.01 & 36.13 & 9.44 & 1.65 & 8.32 & 160.77 & 38.57 & 2.59 & -48.6 & -8.4 \\
\hline 38 & 7.88 & 1.33 & 2.86 & 6.71 & 7.54 & 6.49 & 8.65 & 26.60 & 15.09 & 2.40 & -50.6 & -8.8 \\
\hline 39 & 63.52 & 5.73 & 35.06 & 79.22 & 128.22 & 1.20 & 12.16 & 441.95 & 46.76 & 2.99 & -63.6 & -10.4 \\
\hline 40 & 7.68 & 0.72 & 3.78 & 13.68 & 4.31 & 1.53 & 8.42 & 60.56 & 21.72 & 2.43 & -62.5 & -10.2 \\
\hline SR & 3.18 & 0.66 & 1.02 & 7.62 & 2.49 & 0.61 & 5.36 & 28.55 & 8.99 & 2.56 & -66.2 & -10.7 \\
\hline OR & 5.00 & 1.08 & 1.87 & 8.65 & 4.45 & 0.96 & 6.24 & 33.51 & 8.86 & 2.75 & -59.7 & -9.5 \\
\hline
\end{tabular}

\section{RESUlTS AND DISCUSSION}

The chemical and isotopic compositions of water samples are shown in Tables 1 to 4 and Figs. 1 to 8, where the sample numbers correspond to those in Fig. 1b.
Temperature, EC and ORP profiles of groundwater in the observation well (P3)

In one of the observation wells, Kamizeki (P3 in Fig. $1 \mathrm{~b}$ and Table 3), water temperature, EC, ORP, and $\mathrm{pH}$ were measured as a function of depth (Fig. 2) using a 
Table 3. Sample locality of groundwaters in observation wells. Sample numbers correspond to those in Fig. 1

\begin{tabular}{lcccccccrr}
\hline Sample name & Sampling date & Latitude & Longitude & Depth $(\mathrm{m})$ & Altitude $(\mathrm{m})$ & Temp. $\left({ }^{\circ} \mathrm{C}\right)$ & $\mathrm{pH}$ & $\mathrm{EC}(\mathrm{mS} / \mathrm{m})$ & ORP $(\mathrm{mV}) \mathrm{Pt} *$ \\
\hline P1 & 2011.8 .10 & $36^{\circ} 44^{\prime} 48.43^{\prime \prime}$ & $137^{\circ} 5^{\prime} 26.44^{\prime \prime}$ & 30 & 3 & 20.1 & 8.06 & 14.8 & -110 \\
P2-0 & 2011.8 .10 & $36^{\circ} 46^{\prime} 13.64^{\prime \prime}$ & $137^{\circ} 2^{\prime} 17.78^{\prime \prime}$ & 0 & 3 & 37.8 & 8.92 & 44.3 & 28 \\
P2-33 & 2011.8 .10 & $36^{\circ} 46^{\prime} 13.64^{\prime \prime}$ & $137^{\circ} 2^{\prime} 17.78^{\prime \prime}$ & 33 & 3 & 28.7 & 8.99 & 47.5 & 3 \\
P2-67 & 2011.8 .10 & $36^{\circ} 46^{\prime} 13.64^{\prime \prime}$ & $137^{\circ} 2^{\prime} 17.78^{\prime \prime}$ & 67 & 3 & 25.3 & 9.18 & 48.9 & -80 \\
P2-100 & 2011.8 .10 & $36^{\circ} 46^{\prime} 13.64^{\prime \prime}$ & $137^{\circ} 2^{\prime} 17.78^{\prime \prime}$ & 100 & 3 & 24.9 & 9.16 & 61.0 & -128 \\
P3-0 & 2011.8 .10 & $36^{\circ} 43^{\prime} 44.27^{\prime \prime}$ & $137^{\circ} 0^{\prime} 36.48^{\prime \prime}$ & 0 & 14 & 38.3 & 8.42 & 49.6 & 35 \\
P3-50 & 2011.8 .10 & $36^{\circ} 43^{\prime} 44.27^{\prime \prime}$ & $137^{\circ} 0^{\prime} 36.48^{\prime \prime}$ & 50 & 14 & 25.0 & 8.32 & 50.1 & -172 \\
P3-107 & 2011.8 .10 & $36^{\circ} 43^{\prime} 44.27^{\prime \prime}$ & $137^{\circ} 0^{\prime} 36.48^{\prime \prime}$ & 107 & 14 & 24.7 & 8.11 & 52.7 & -188 \\
P4 & 2011.8 .11 & $36^{\circ} 36^{\prime} 33.07^{\prime \prime}$ & $136^{\circ} 57^{\prime} 54.80^{\prime \prime}$ & 33 & 73 & 19.6 & 7.72 & 16.6 & 85 \\
P5 & 2011.8 .11 & $36^{\circ} 34^{\prime} 43.98^{\prime \prime}$ & $136^{\circ} 53^{\prime} 35.44^{\prime \prime}$ & 33 & 59 & 17.6 & 7.97 & 13.7 & -170 \\
P6 & 2011.8 .11 & $36^{\circ} 38^{\prime} 28.64^{\prime \prime}$ & $136^{\circ} 54^{\prime} 5.06^{\prime \prime}$ & 33 & 40 & 17.2 & 8.65 & 8.7 & 25 \\
P7 & 2011.8 .11 & $36^{\circ} 42^{\prime} 3.25^{\prime \prime}$ & $136^{\circ} 56^{\prime} 16.90^{\prime \prime}$ & 0 & 19 & 31.8 & 8.37 & 15.8 & 37 \\
P8 & 2011.9 .13 & $36^{\circ} 39^{\prime} 10.85^{\prime \prime}$ & $136^{\circ} 56^{\prime} 36.85^{\prime \prime}$ & 20 & 42 & 22.7 & 8.85 & 8.7 & 87 \\
P9 & 2011.9 .9 & $36^{\circ} 42^{\prime} 47.96^{\prime \prime}$ & $137^{\circ} 0^{\prime} 34.67^{\prime \prime}$ & 50 & 13 & 13.8 & 8.28 & 11.4 & 76 \\
\hline
\end{tabular}

* Measured with a Pt electrode.

Table 4. Chemical and stable isotopic compositions of groundwaters in observation wells at Sho River fan area. Sample numbers correspond to those in Fig. 1

\begin{tabular}{|c|c|c|c|c|c|c|c|c|c|c|c|c|}
\hline \multirow[t]{2}{*}{ Sample name } & $\mathrm{Na}^{+}$ & $\mathrm{K}^{+}$ & $\mathrm{Mg}^{2+}$ & $\mathrm{Ca}^{2+}$ & $\mathrm{Cl}^{-}$ & $\mathrm{NO}_{3}^{-}$ & $\mathrm{SO}_{4}{ }^{2-}$ & $\mathrm{HCO}_{3}^{-}$ & $\mathrm{SiO}_{2}$ & \multirow{2}{*}{$\begin{array}{c}\mathrm{Fe} \\
\mu \mathrm{g} / \mathrm{L}\end{array}$} & $\delta \mathrm{D}$ & $\delta^{18} \mathrm{O}$ \\
\hline & \multicolumn{9}{|c|}{$\mathrm{mg} / \mathrm{L}$} & & \multicolumn{2}{|c|}{$\%$} \\
\hline $\mathrm{P} 1$ & 14.28 & 1.66 & 5.35 & 7.41 & 8.70 & 0.000 & 0.96 & 68.63 & 6.35 & 69.83 & -48.4 & -7.2 \\
\hline $\mathrm{P} 2-0$ & 94.50 & 6.40 & 1.50 & 5.40 & 79.00 & 0.000 & 1.10 & 138.78 & 2.00 & 4.26 & -45.1 & -7.3 \\
\hline $\mathrm{P} 2-33$ & 97.20 & 5.30 & 1.60 & 6.30 & 82.00 & 0.000 & 1.00 & 131.15 & 3.90 & 10.76 & -51.4 & -8.2 \\
\hline P2-67 & 102.50 & 6.00 & 1.50 & 5.75 & 97.00 & 0.000 & 0.75 & 126.27 & 17.40 & 226.28 & -52.3 & -8.4 \\
\hline P2-100 & 235.50 & 12.00 & 5.00 & 16.50 & 317.50 & 0.000 & 0.50 & 129.32 & 38.20 & 237.32 & -55.6 & -9.0 \\
\hline P3-0 & 55.70 & 3.10 & 29.20 & 15.40 & 20.40 & 0.000 & 0.00 & 277.55 & 11.40 & 2.51 & -51.0 & -8.2 \\
\hline $\mathrm{P} 3-50$ & 50.00 & 2.60 & 29.00 & 29.80 & 19.00 & 0.000 & 0.00 & 308.05 & 19.30 & 23.02 & -54.6 & -8.7 \\
\hline P3-107 & 46.30 & 2.60 & 27.70 & 41.50 & 17.60 & 0.000 & 0.00 & 326.96 & 32.60 & 78.80 & -57.1 & -9.1 \\
\hline P4 & 5.73 & 0.95 & 3.08 & 25.30 & 6.23 & 0.050 & 3.20 & 94.55 & 10.80 & 2.60 & -57.4 & -9.7 \\
\hline P5 & 10.73 & 1.25 & 2.30 & 10.28 & 4.63 & 0.000 & 1.10 & 62.22 & 10.45 & 3.26 & -46.9 & -8.5 \\
\hline P6 & 8.33 & 0.95 & 0.65 & 7.83 & 8.85 & 0.000 & 0.13 & 37.21 & 0.90 & 2.57 & -50.6 & -9.5 \\
\hline P7 & 9.98 & 1.38 & 5.33 & 11.33 & 12.58 & 0.025 & 0.18 & 63.44 & 6.95 & 55.50 & -42.9 & -6.5 \\
\hline P8 & 7.26 & 3.81 & 0.59 & 13.57 & 1.58 & 0.168 & 6.33 & 58.56 & 12.40 & 274.33 & -55.0 & -8.9 \\
\hline P9 & 4.59 & 0.88 & 0.79 & 6.54 & 4.62 & 0.023 & 0.04 & 28.67 & 0.30 & 9.13 & -55.7 & -9.3 \\
\hline
\end{tabular}

water monitoring system (MP-TROLL9000). It is noted that the water temperature is almost constant at $15^{\circ} \mathrm{C}$ between 10 to $80 \mathrm{~m}$ and increases monotonously up to $23^{\circ} \mathrm{C}$ at $220 \mathrm{~m}$ depth. The EC value normalized at $25^{\circ} \mathrm{C}$ decreases from 56 to $42 \mathrm{mS} / \mathrm{m}$ at the surface zone and increases up to $65 \mathrm{mS} / \mathrm{m}$ with increasing depth (Fig. 2). The ORP increases from -220 to $-100 \mathrm{mV}$ with depth. A strainer tube installed between 160 to $175 \mathrm{~m}$ depth allowed groundwater to flow into the well. The EC and temperature profiles show that the groundwater can be divided into 3 layers; 10 to $60 \mathrm{~m}, 60$ to $160 \mathrm{~m}$ and the deepest layer except for the surface layer (surface to $10 \mathrm{~m}$ ).
Groundwater samples were collected at the depth of 0 (surface), 50, and $100 \mathrm{~m}$ using a water sampler (Table 3). If there is only one strainer in the deep part of the well, water chemistry would have been the same at all depths. The observed variations imply that the water with different EC and temperature mixes above the strainer depth due to casing break.

The most important characteristic of groundwater profile in the observation well is the high geothermal gradient of $c a .6^{\circ} \mathrm{C} / 100 \mathrm{~m}$ below a depth of $80 \mathrm{~m}$. The high geothermal gradient suggests existence of a high temperature heat source exists under the study area. 


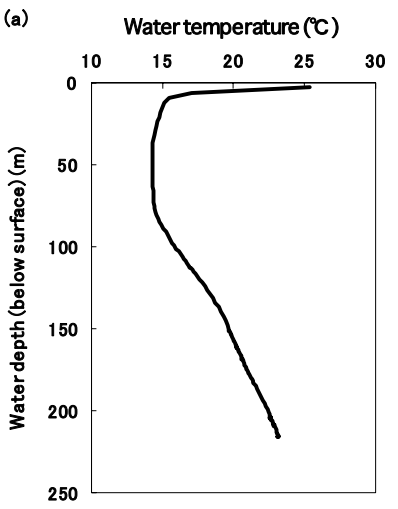

(b)

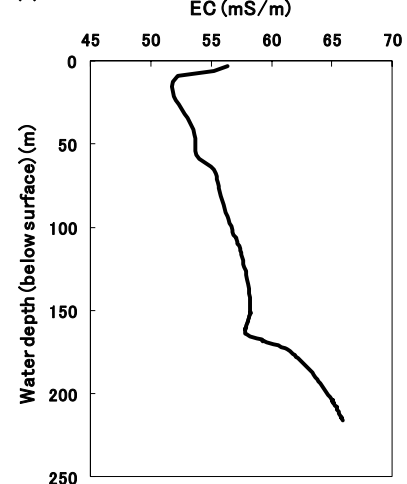

(c)

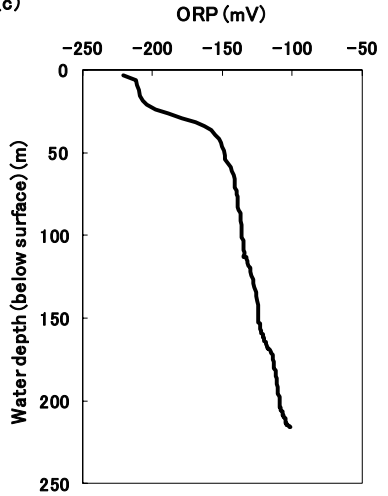

(d)

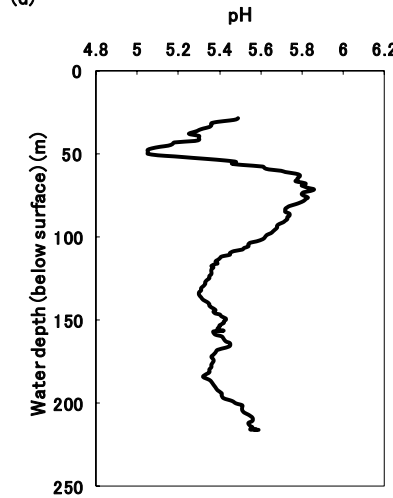

Fig. 2. Variation with depth in water temperature (a), EC (b), ORP $(c)$ and $p H(d)$ of groundwater in the Kamizaki observation well $P 3$.

\section{$\delta D$ and $\delta^{18} O$ values of water samples}

The $\delta \mathrm{D}$ and $\delta^{18} \mathrm{O}$ values of shallow groundwaters vary from -46.5 to $-64.6 \%$ and from -8.1 to $-10.6 \%$, respectively (Table 2), whereas those of well waters are relatively high $\left(\delta \mathrm{D}:-46.9 \sim-57.1 \%\right.$ and $\delta^{18} \mathrm{O}:-7.2 \sim-9.3 \%$; Table 4 and Fig. 3). Water sample from Sho river has the lowest $\delta \mathrm{D}(-66.2 \% o)$ and $\delta^{18} \mathrm{O}(-10.7 \%$ ) values (Fig. 3) of the samples. Most of the water samples plot along a meteoric water line defined as $\delta \mathrm{D}=8 \delta^{18} \mathrm{O}+d$ (Craig, 1961; Dansgaard, 1964) where $d$ indicates the $Y$-intercept. Two meteoric water lines in Fig. 3 were quoted from Satake et al. (1984), where d is 10 in summer and 30 in winter for precipitation in Toyama City, $20 \mathrm{~km}$ east of the Sho river fan. The $\delta \mathrm{D}$ and $\delta^{18} \mathrm{O}$ values of Toyama precipitation were monitored from 1982 to 1995 as shown in Table 5. The range of variation is reproduced as an open box in Fig. 3 .

The $\delta \mathrm{D}$ and $\delta^{18} \mathrm{O}$ values of river waters were periodically analyzed between 1980 and 1982 (Mizutani and Oda, 1983) of which variations for the Sho and Oyabe rivers are shown in Fig. 3 as solid and open circles, respectively. The averaged $\delta \mathrm{D}$ and $\delta^{18} \mathrm{O}$ values are $-63.3 \%$ and $-10.4 \%$ o for the Sho river and $-55.2 \%$ and $-9.4 \%$ for the Oyabe

Table 5. $\delta D$ and $\delta^{18} O$ values of Precipitation at Toyama City

\begin{tabular}{cccc}
\hline Year & $\begin{array}{c}\text { Precipitation } \\
(\mathrm{mm})\end{array}$ & $\begin{array}{c}\delta \mathrm{D} \\
(\% \mathrm{o})\end{array}$ & $\begin{array}{c}\delta^{18} \mathrm{O} \\
(\% \circ)\end{array}$ \\
\hline 1982 & 1555 & -54 & -8.8 \\
1983 & 2871 & -54 & -9.3 \\
1984 & 2224 & -50 & -9.5 \\
1985 & 2969 & -56 & -9.4 \\
1986 & - & - & - \\
1987 & 1281 & -43 & -7.4 \\
1988 & 1731 & -52 & -8.8 \\
1989 & - & - & - \\
1990 & - & - & - \\
1991 & 1984 & -54 & -8.8 \\
1992 & 1816 & -48 & -8.6 \\
1993 & 2213 & -54 & -8.0 \\
1994 & 1411 & -37 & -7.1 \\
1995 & 1756 & -48 & -8.3 \\
& & & \\
Averaged* & 1982 & -51 & -8.6 \\
\hline
\end{tabular}

*Weighted mean averaged values of $\delta D$ and $\delta^{18} O$ Data reproduced from Satake et al. (1984).

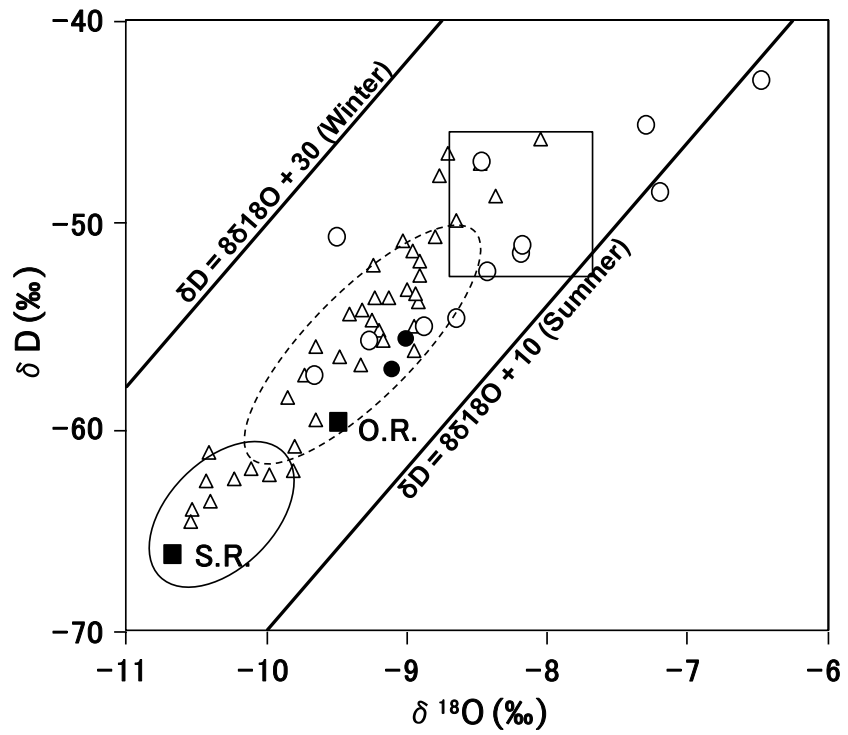

Fig. 3. $\delta D$ vs. $\delta^{18} O$ relationship of water samples in the Sho river fan. Open triangles indicate the shallow groundwater, solid squares the Sho and Oyabe rivers, open circles the observation well water (above $80 \mathrm{~m}$ ), and solid circles the observation well water (below $80 \mathrm{~m}$ ). The box indicates the range of isotopic variations for precipitation in Toyama city (averaged data for 5 years since 1991 measured by Satake, unpublished). Solid and broken circles correspond to the range of variation for the Sho (S.R.) and Oyabe (O.R.) river waters (data from Mizutani and Oda, 1983). Two solid lines indicate the local meteoric water lines in the winter and summer at Toyama City (Satake et al., 1984). 


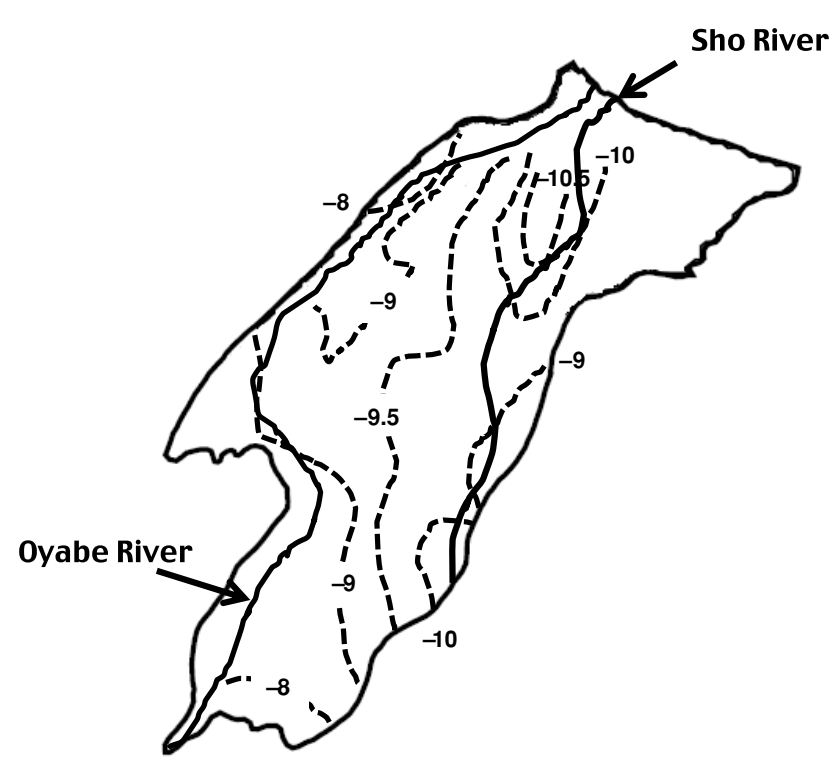

Fig. 4. Distribution of $\delta^{18} \mathrm{O}$ values of shallow groundwater collected in 2011 in the Sho river fan. river, respectively (Mizutani and Oda, 1983), almost similar to the present results (Table 2). In Fig. 3, the range of variation in $\delta \mathrm{D}$ and $\delta^{18} \mathrm{O}$ values of the Sho river (solid circle in Fig. 3) is smaller than that of the Oyabe river (broken circle). As mentioned before, the Sho river originates from high altitude, whereas the Oyabe river comes from lower mountains. These geographical differences caused large variations in $\delta \mathrm{D}$ and $\delta^{18} \mathrm{O}$ values of the two rivers due to a large difference in the isotopic values of the source precipitation.

The observed $\delta \mathrm{D}$ and $\delta^{18} \mathrm{O}$ values of shallow groundwaters in the Sho river fan are consistent with the ranges of averaged precipitation and river waters of the area (Fig. 3). The contour of $\delta^{18} \mathrm{O}$ distribution are shown in Fig. 4. We can see that two groundwaters derived from the Sho and Oyabe rivers infiltrate to the central part of the fan. Along the Oyabe river, the $\delta^{18} \mathrm{O}$ values of groundwater are -8.5 to $-9 \%$ which are higher than the river water $\delta^{18} \mathrm{O}$ value $(-9.5 \%$ ). This means that shallow groundwaters around the Oyabe river are a mixture of river water and precipitation. In contrast, the $\delta^{18} \mathrm{O}$ values of shallow groundwater along the Sho river are similar to those of river water $(-10.7 \%$ o). These results show that the Sho river water infiltrates at the top of the fan and discharges at the end of the fan. Though the Oyabe river

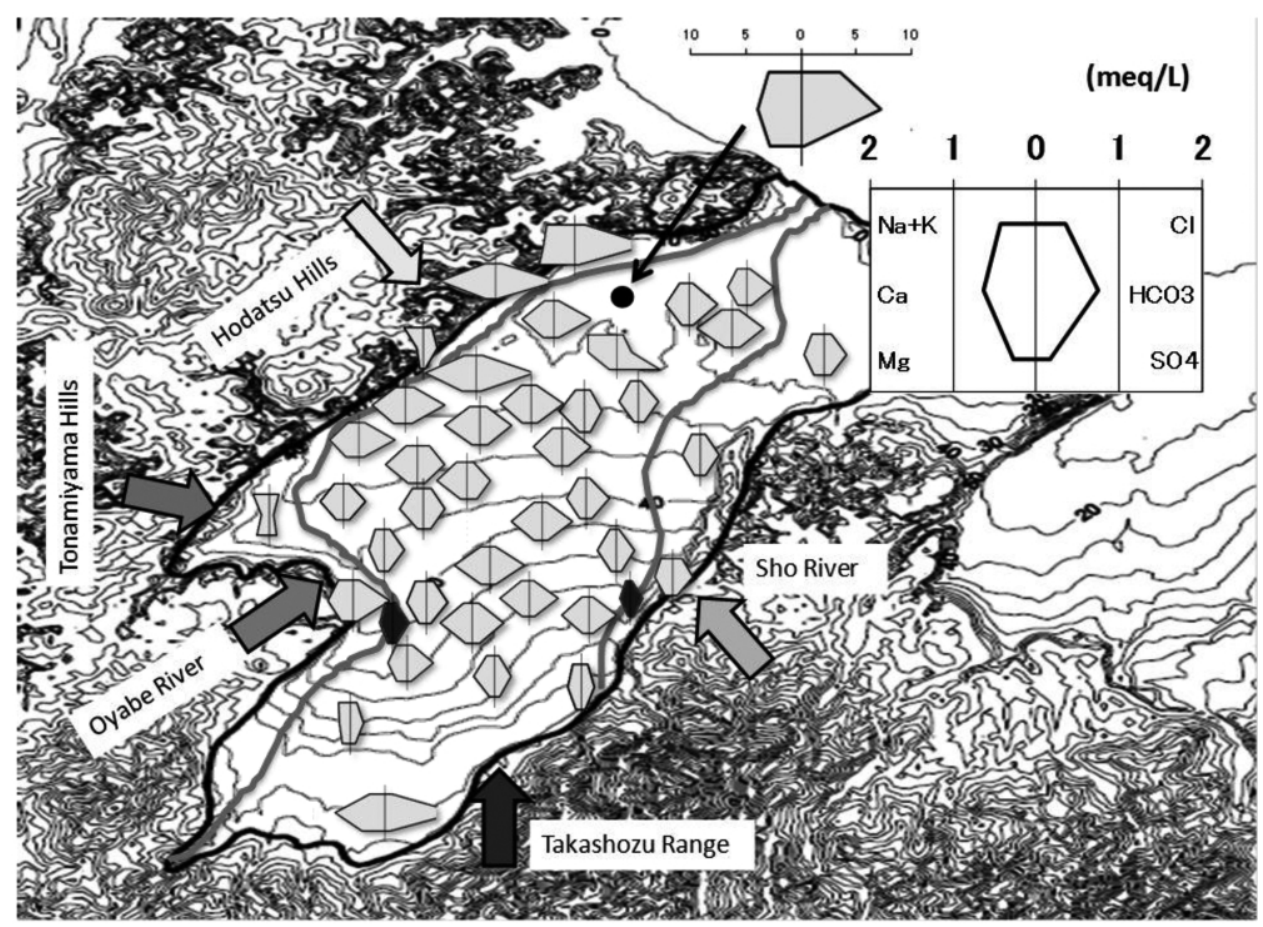

Fig. 5. Distribution of the chemical type of shallow groundwaters and river waters in the Sho river fan. The chemical type is shown by the inserted hexa-diagrams. The solid hexagons correspond to the chemical type of the river waters. Solid circle shows the groundwater No. 39 that has a high EC value. The five arrows in the figure mean the water sources of groundwaters in the Sho river fan as demonstrated by Mizutani and Oda (1983). The contour is given in $10 \mathrm{~m}$ interval. The highest altitude is $150 \mathrm{~m}$ a.s.l. at the southernmost end of the fun. 


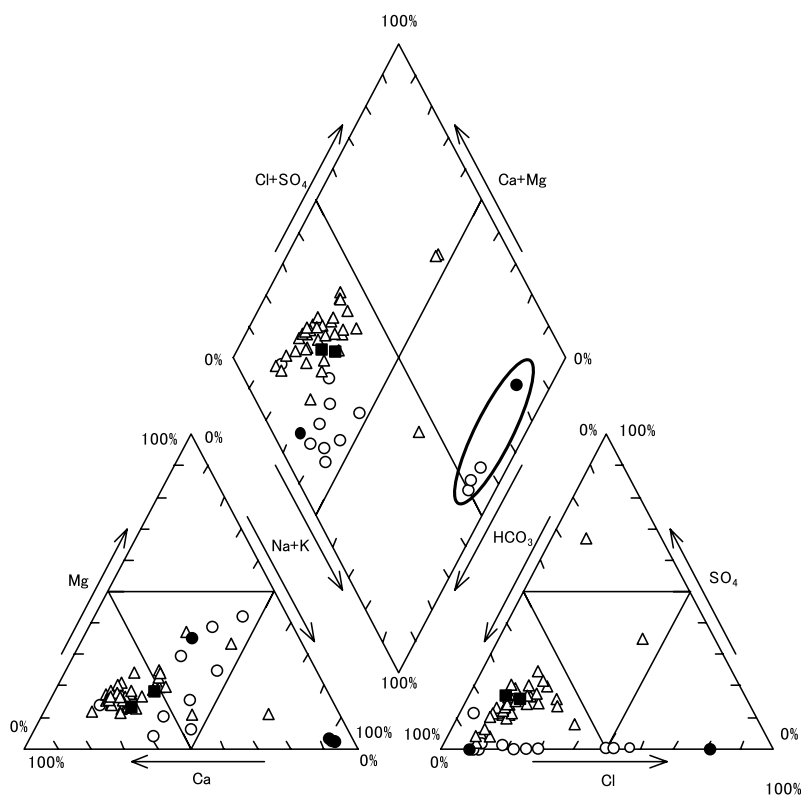

Fig. 6. The Piper diagram for water samples in the Sho river fan. Open triangles indicate the shallow groundwater, solid squares the Sho and Oyabe rivers, open circles the observation well water (above $80 \mathrm{~m}$ ), and solid circles the observation well water (below $80 \mathrm{~m}$ ). Solid circle shows groundwater in well $\mathrm{P3}$. The water was observed to change from the $\mathrm{Na}-\mathrm{HCO}_{3}$ to $\mathrm{NaCl}$ types with increasing depth.

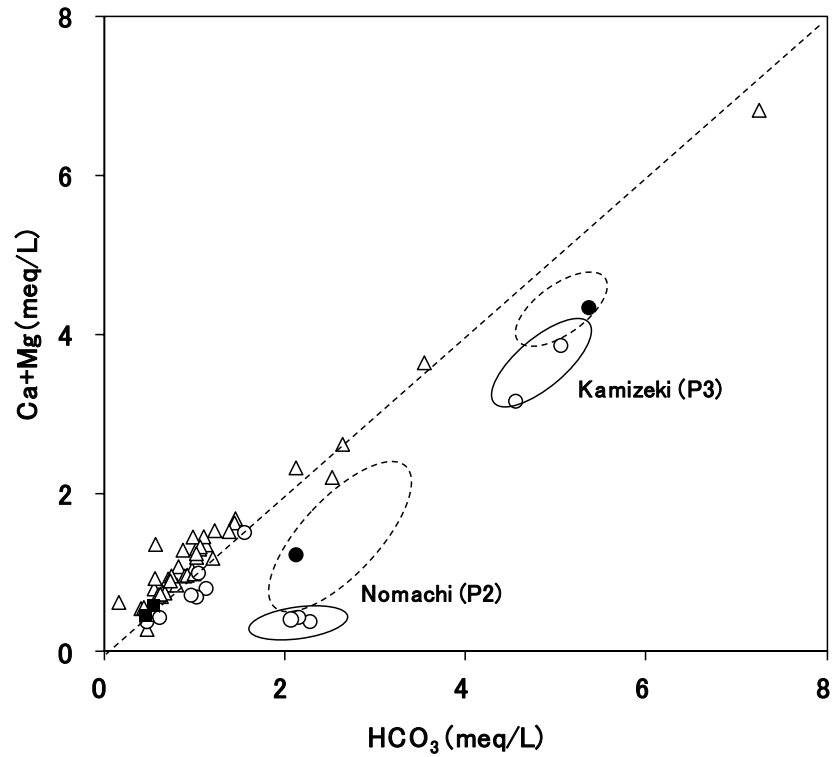

Fig. 7. Relationship between $\mathrm{Ca}+\mathrm{Mg}$ and $\mathrm{HCO}_{3}$ concentrations of the Sho river fan waters. Open triangles indicate the shallow groundwater, solid squares the Sho and Oyabe rivers, open circles the observation well water (above $80 \mathrm{~m}$ ), and solid circles the observation well water (below $80 \mathrm{~m}$ ). Broken line corresponds to dolomite dissolution. Solid and broken circles show the range of variation for the shallow and deep groundwaters, respectively.
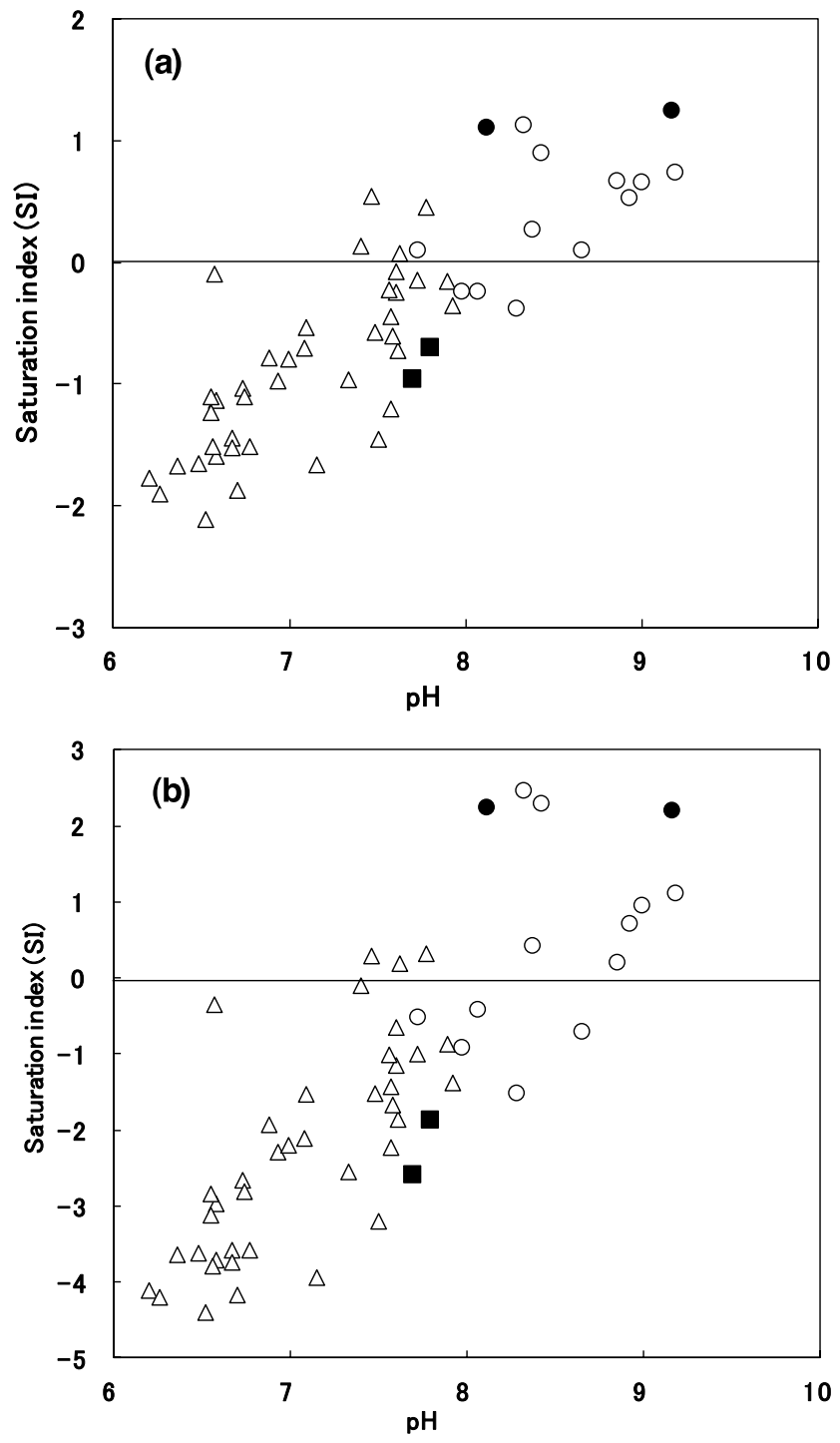

Fig. 8. Saturation indexof (a) calcite and (b) dolomite as a function of $\mathrm{pH}$. Open triangles indicate the shallow groundwater, solid squares the Sho and Oyabe rivers, open circles the observation well water (above $80 \mathrm{~m}$ ), and solid circles the observation well water (below $80 \mathrm{~m}$ ).

flows near the Nanto city (see Fig. $1 \mathrm{~b}$ ), $\delta^{18} \mathrm{O}$ value of groundwater around Nanto is almost $-8 \%$ (Fig. 4). The groundwater of this region is strongly influenced by the precipitation.

\section{Chemical compositions}

All shallow groundwaters have weakly acid to alkaline with $\mathrm{pH}$ values ranging from 6.20 to 7.89 (Table 1). In contrast, those of groundwaters from the observation wells (shallow water above $-80 \mathrm{~m}$ and deep below -80 $\mathrm{m}$ ) are relatively high to be 7.72 to 9.18 (Table 3 ). The EC values are 8.9 to $37.1 \mathrm{mS} / \mathrm{m}$ except for sample No. 39 


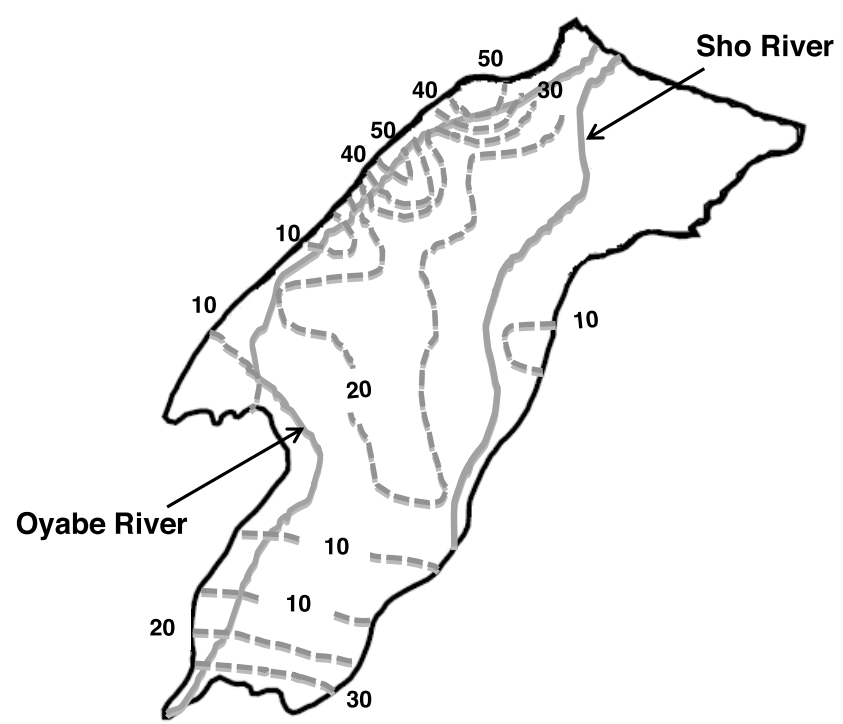

Fig. 9. Distibution of Ca concentration $(\mathrm{mg} / \mathrm{L})$ in shallow groundwaters in the Sho river fan.

(solid circle in Fig. $5 . \mathrm{EC}=88 \mathrm{mS} / \mathrm{m}$ ). These results are almost similar to those reported by Miyamoto (2000). The EC values of groundwaters in the observation well vary from 8.7 to $52.7 \mathrm{mS} / \mathrm{m}$ (Table 3), relatively high compared to shallow groundwaters. In the upper part of the fan, the shape of the hexa-diagrams for shallow groundwaters is similar to that of river waters (solid hexagons in Fig. 5). This suggests that both river waters infiltrate into the fan in the upper area. Shallow groundwaters in the central and lower part of the fan are characterized by high $\mathrm{Ca}$ and $\mathrm{HCO}_{3}$ concentrations (Fig. 5). Furthermore, the flow of groundwater in the fan can be divided into five water systems (arrows in Fig. 5) based on the chemical compositions as suggested by Mizutani and Oda (1983).

Figure 6 shows a trilinear diagram of the chemical compositions of all water samples. Shallow groundwaters and river waters are mainly of a $\mathrm{Ca}-\mathrm{HCO}_{3}$ type with a few exceptions. Water samples from observation wells plot widely in Fig. 6. The EC values of groundwaters in well P3 (solid circle in Fig. 6) increase with increasing depth (Fig. 2) with the associated chemical change from $\mathrm{Na}-\mathrm{HCO}_{3}$ to $\mathrm{NaCl}$ types. It is thought that seawater flows into the deep part.

The $\mathrm{Ca}+\mathrm{Mg}$ concentrations of water samples increase linearly with increasing $\mathrm{HCO}_{3}$ as shown in Fig. 7, where the broken line indicates dolomite dissolution. The linearity implies that the shallow groundwaters become enriched in $\mathrm{Ca}, \mathrm{Mg}$, and $\mathrm{HCO}_{3}$ due to dissolution of dolomite. In contrast, the groundwaters in the observation wells are plotted below the broken line. In Fig. 7, solid
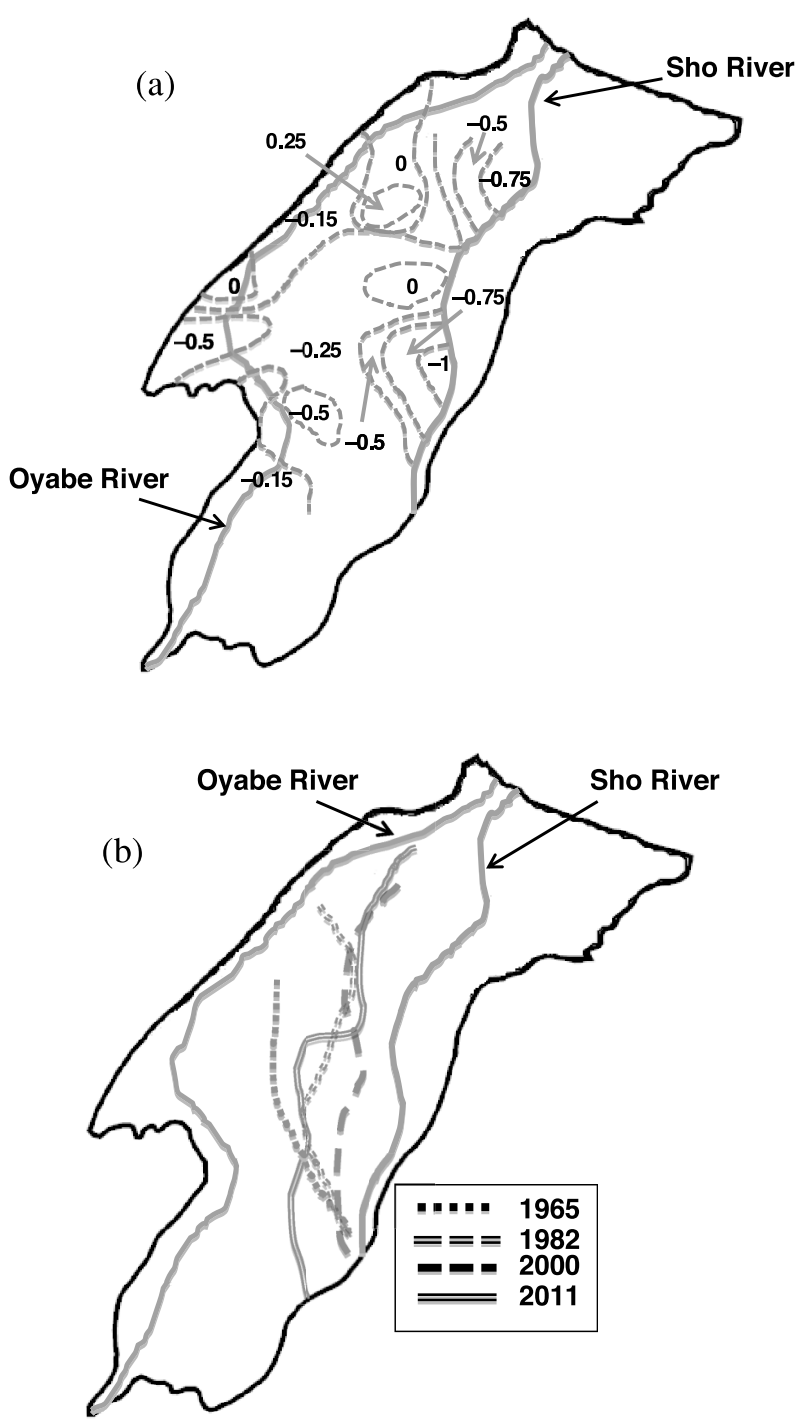

Fig. 10. (a) Distibution of $\delta^{18} \mathrm{O}$ difference in shallow groundwaters between 2000 and 2011. The 2000 data were quoted from Miyamoto (2000). (b) Temporal change of the boundary between the Sho and Oyabe water systems from 1965 to present (after Mizutani and Oda, 1983 for data in 1965 and 1983, and Miyamoto, 2000 for 2000).

and broken circles show the ranges of observed chemical variations of the shallow (above $80 \mathrm{~m}$ ) and deep (below $80 \mathrm{~m}$ ) groundwater in wells P2 (Nomachi) and P3 (Kamizeki). Our shallow and deep samples are plotted as open and solid circles, respectively. Sodium concentration in groundwaters from the observation wells is higher than that in shallow groundwater in the Sho river fan and correlates with $\mathrm{K}$ concentration (Tables 2 and 4 ). Groundwaters in the observation well are enriched in $\mathrm{Na}$ and $\mathrm{K}$ and depleted in $\mathrm{Ca}$ through ion exchange with clay minerals that underlie the northern part of the fan. The 


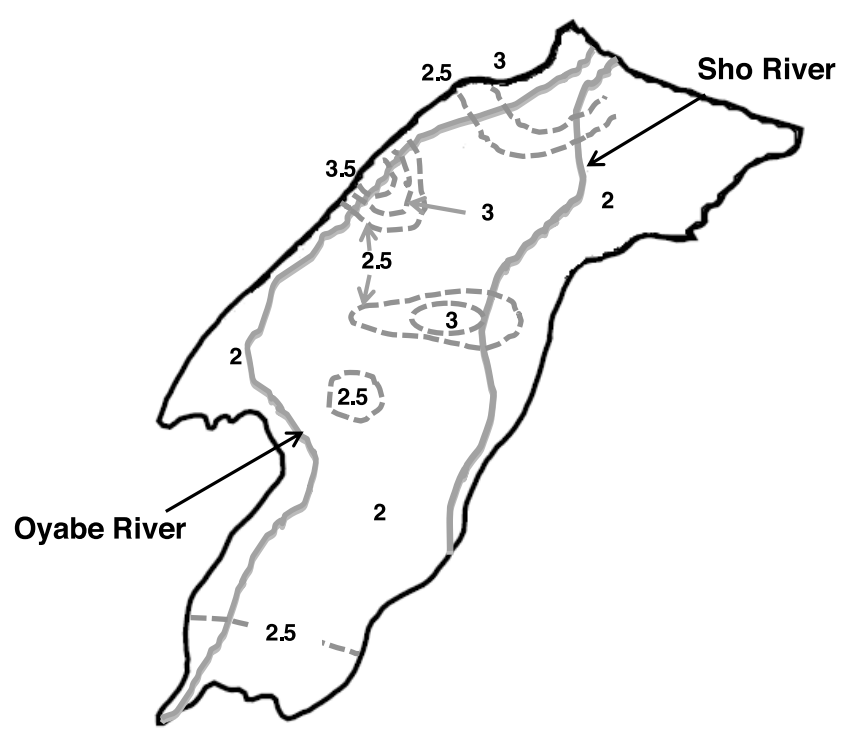

Fig. 11. Distibution of Fe concentration $(\mu g / L)$ in shallow groundwaters in the Sho river fan.

$\mathrm{pH}$ values of deep groundwater also become higher than the shallow groundwater.

The saturation indices of calcite $\left(\mathrm{CaCO}_{3}\right)$ and dolomite $\left(\mathrm{CaMg}\left(\mathrm{CO}_{3}\right)\right)$ were calculated to discuss the source of $\mathrm{Ca}$ and $\mathrm{Mg}$ and also to examine the possibility of precipitation of such minerals during heat exchange by GeoHP. Saturation Index (S.I.) is defined by;

$$
\text { S.I. (Saturation Index })=\log (Q / K)
$$

where $Q$ and $K$ are, respectively, activity product $\left(a_{\mathrm{Ca} 2+} * a_{\mathrm{CO} 32-}\right.$ in the case of calcite) and solubility product $\left(\left(\mathrm{Ca}^{2+}\right)\left(\mathrm{CO}_{3}{ }^{2-}\right)\right)$ of minerals. If S.I. $>0$, the fluid is saturated with respect to the minerals. The chemical code PHREEQC (Parkhurst and Appelo, 1999) was used in this study. The obtained S.I. values of water samples are plotted against $\mathrm{pH}$ as shown in Fig. 8. The S.I. of calcite and dolomite increases with increasing $\mathrm{pH}$, and above $\mathrm{pH}$, groundwater should be saturated with respect to these minerals. Groundwaters in the observation wells are oversaturated because of their high $\mathrm{pH}$ and high $\mathrm{Ca}$ and $\mathrm{HCO}_{3}$ concentrations. The S.I. of gypsum $\left(\mathrm{CaSO}_{4}\right)$ was also calculated, but it was always below unity, or gypsum undersaturated. These results imply that shallow groundwater derives essentially from river waters that infiltrated into underground and enriched in $\mathrm{Ca}$ and $\mathrm{HCO}_{3}$ due to dissolution of carbonate minerals such as calcite and dolomite. Carbonate rocks are observed in the several formations in the Sho river fan (Sumi et al., 1989). The hydrolysis and dissolution reactions of limestone and dolomite to form dissolved bicarbonate, calcium and
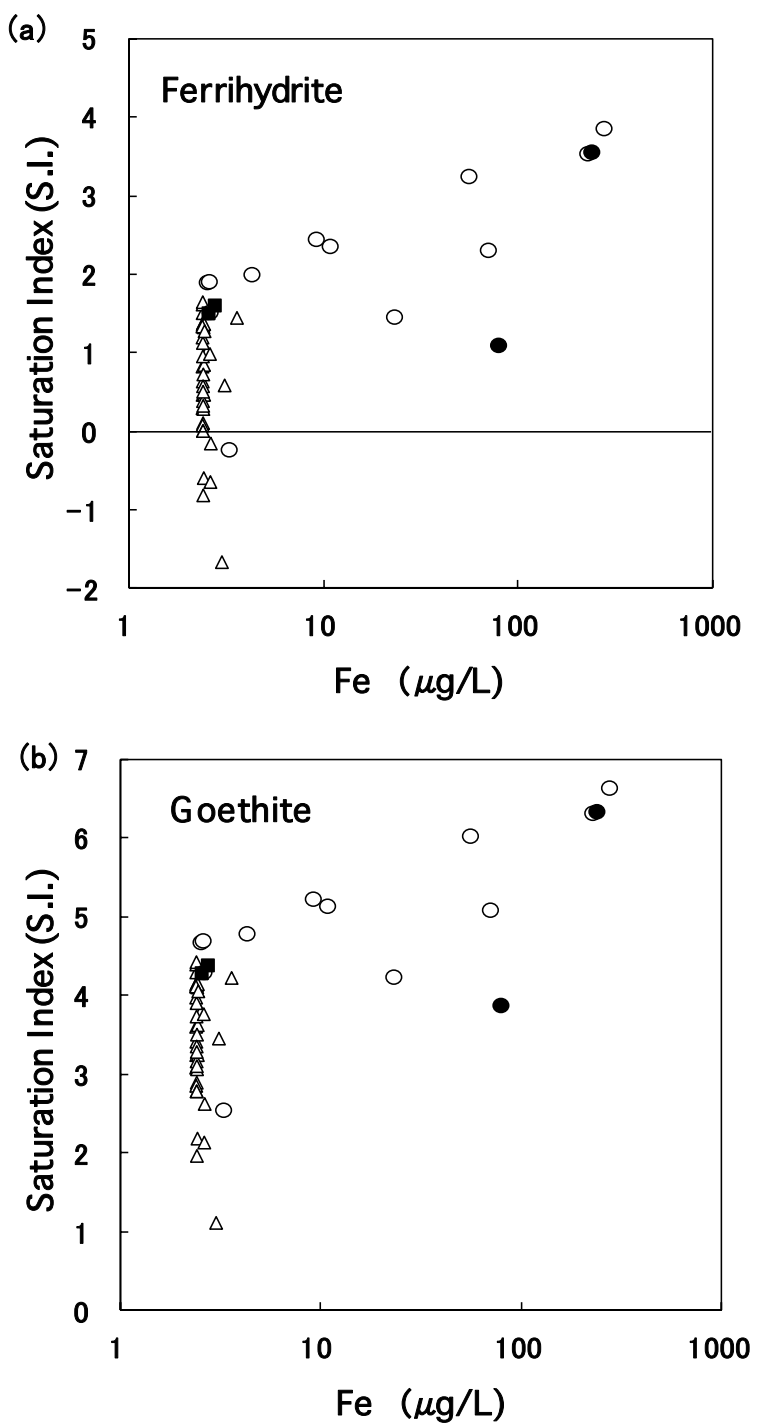

Fig. 12. Saturation index of (a) ferrihydrite $\left(\mathrm{Fe}(\mathrm{OH})_{3}\right)$ and (b) goethite ( $\mathrm{FeOOH}$ ) as a function of $\mathrm{Fe}$ concentration. Open triangles indicate the shallow groundwater, solid squares the Sho and Oyabe rivers, open circles the observation well water (above $80 \mathrm{~m}$ ), and solid circles the observation well water (below $80 \mathrm{~m}$ ).

magnesium ions can be described as (Appelo and Postma, 2005):

$\mathrm{CaCO}_{3}+\mathrm{H}_{2} \mathrm{O}=\mathrm{Ca}^{2+}+\mathrm{HCO}_{3}^{-}+\mathrm{OH}^{-}$

$\mathrm{CaMg}\left(\mathrm{CO}_{3}\right)+2 \mathrm{H}_{2} \mathrm{O}=\mathrm{Ca}^{2+}+\mathrm{Mg}^{2+}+2 \mathrm{HCO}_{3}^{-}+2 \mathrm{OH}^{-}$

$\mathrm{CO}_{2}+\mathrm{H}_{2} \mathrm{O}=\mathrm{HCO}_{3}^{-}+\mathrm{H}^{+}$.

If $\mathrm{CO}_{2}$ is available from the atmosphere and/or decom- 


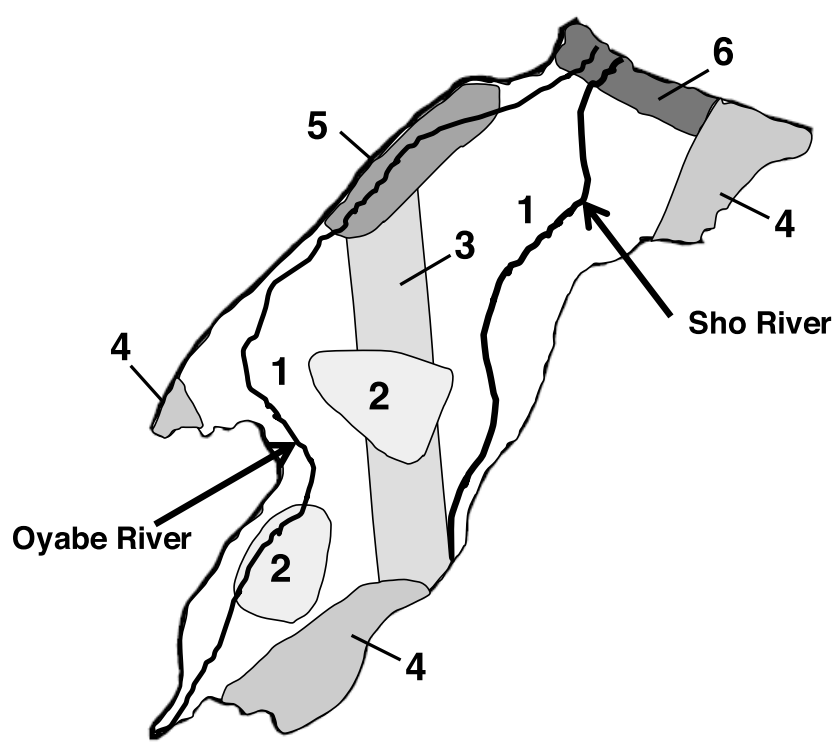

Fig. 13. A map showing the areas where an open type or closed type Geo-HP is recommended in the Sho river fan (See text). 1: Open type Geo-HP can be used, but iron scale may cause a problem. 2: Open type Geo-HP can be used without any scaling problem. 3: Open type Geo-HP can be used with necessary of monitoring the change of water flow. 4: Closed type Geo-HP is recommended. 5: Closed type Geo-HP is recommended. Groundwaters with high EC value may induce calcite scaling. 6: Not recommended to any type of Geo-HP, because of high salinity from sea water contamination.

position of organic matter, the equations are;

$$
\begin{aligned}
& \mathrm{CaCO}_{3}+\mathrm{H}_{2} \mathrm{O}+\mathrm{CO}_{2}=\mathrm{Ca}^{2+}+2 \mathrm{HCO}_{3}^{-} \\
& \mathrm{CaMg}\left(\mathrm{CO}_{3}\right)+\mathrm{H}_{2} \mathrm{O}+\mathrm{CO}_{2}=\mathrm{Ca}^{2+}+\mathrm{Mg}^{2+}+2 \mathrm{HCO}_{3}^{-} .
\end{aligned}
$$

Calcium and magnesium ions in the groundwaters from alluvial fans are often correlated, and these components are considered to derive from dissolution of carbonate minerals (e.g., Hidalgo and Cruz-Sanjulian, 2001; Chkirbene et al., 2009). These rocks are the most likely source of $\mathrm{Ca}$ and $\mathrm{Mg}$ in the groundwaters.

Figure 9 is a contour map of $\mathrm{Ca}$ distribution in the shallow groundwaters. It is noted that the $20 \mathrm{mg} / \mathrm{L}$ contour is widely found in the central part of the fan. In the Sho river fan, basement rocks form depression in the central part of the fan and its minimum depth is estimated to be $-300 \mathrm{~m}$ (Miyamoto, 2000). This means that part of shallow groundwater infiltrates into deeper part of the central part of the fan and discharges in the northern part of the fan as confined groundwater. During this process, shallow groundwaters acquire $\mathrm{Na}, \mathrm{K}$ and $\mathrm{HCO}_{3}$ as noted by Miyamoto (2000).
Water quality usable for geothermal heat pump

Water movement is one of the key parameters for utilizing a geothermal heat pump. Figure 10a shows the difference in $\delta^{18} \mathrm{O}$ values of groundwaters between 2000 (Miyamoto, 2000) and 2011. It indicates that most shallow groundwaters in 2011 are depleted in $\delta^{18} \mathrm{O}$ up to $0.75 \%$ compared to those in 2000 . This means that contribution of the Sho river water into groundwater increased over time. Figure 10b shows the boundary between the groundwater originated from the Sho and Oyabe rivers. The boundaries were drawn using the $\delta^{18} \mathrm{O}$ distribution, water level and $\mathrm{pH}$ (the 1965 and 1982 boundaries were taken from Mizutani and Oda (1983)). It is seen that the 1965 boundary was located in the western part of the fan, whereas the 2000 boundary shifted eastward. The 2011 boundary stayed almost the same in the northern part of the fan, but it shifted slightly westward in the southern part. Mizutani and Oda (1983) demonstrated that the Sho river water infiltrated into the main part of the fan. They also stated that the recharge area moved from the top to end of the fan because of reduction in flow rate and lowering of the riverbed. The 2011 boundary moved to the west in the top of the fan as stated above. It is interpreted that the amount of water infiltration from the Sho river increased in recent 10 years.

The quality of groundwater is a key parameter for geothermal heat pump. The EC values of shallow groundwaters were 8.8 to $37.1 \mathrm{mS} / \mathrm{m}$ and almost the same or higher than those of river waters $(8.2$ and $13.7 \mathrm{mS} / \mathrm{m})$. Saturation index calculations indicate that most of the shallow groundwaters are undersaturated with respect to calcite at $15^{\circ} \mathrm{C}$ except for 4 samples (Fig. 8). Calcite may precipitate in the system during heat exchange in heat pump for the 4 groundwaters. Groundwaters may be heated up to $40^{\circ} \mathrm{C}$ in heat exchanger in summer. The SI at this temperature is -0.2 , so some more shallow water may be saturated with calcite. However, the majority of water is still undersaturated. This suggests that scaling by calcite deposition will not be a problem in the study area.

Dissolved iron is a potential component of scaling during heat pumping. Iron in groundwater under reducing condition generally exists as ferrous ion $\left(\mathrm{Fe}^{2+}\right)$ and is oxidized by atmospheric $\mathrm{O}_{2}$ to ferric ion $\left(\mathrm{Fe}^{3+}\right)$ at the surface. Ferric ion will precipitate in the well and piping. Ferrihydrite $\left(\mathrm{Fe}(\mathrm{OH})_{3}\right)$ and goethite $(\mathrm{FeOOH})$ are commonly observed in the scale of groundwater wells (e.g., Schwertmann and Murad, 1983; Charette and Sholkovitz, 2002). In this study, iron concentrations in waters were analyzed (Tables 2,4 ). They vary from 2.4 to $3.6 \mu \mathrm{g} / \mathrm{L}$ (Fig. 11) and 2.6 to $274 \mu \mathrm{g} / \mathrm{L}$ for shallow and deep groundwaters, respectively. Saturation indices of ferrihydrite $\left(\mathrm{Fe}(\mathrm{OH})_{3}\right)$ and goethite $(\mathrm{FeOOH})$ were calculated from the observed ORP values and the chemical compositions of water samples (Figs. 12a and b). In this 
calculation the PHREEQC code was used where the observed ORP values were used for the oxidation-reduction potential $(\mathrm{Pe})$. Most of the shallow groundwaters are saturated with respect to ferrihydrite (except for 6 samples) and goethite. Part of iron in the groundwater will precipitate in the heat exchanger during heat pumping. Kushita et al. (2012) has tested an open-type heat pump at the Nanto City (Fig. 1b) for one year. They compared the power requirement for the first and second floors of a house where the test was performed. Cool air was supplied only to the first floor from the heat pump. It was found that electric consumption was saved to $60 \%$ in the first floor. After this test, a small amount of brown colored deposit was observed in the heat exchanger after 1 year use. The XRF analysis indicated that it contained iron as a major component. This iron precipitate is thought to be come from corrosion of iron casing pipe of the well, but precipitation of iron from groundwater cannot be ruled out. These results demonstrated that groundwater with low $\mathrm{Fe}^{2+}$ concentration and low ORP can be used for an opentype heat pump. In contrast, groundwaters with high ORP need to pay attention for the iron oxide precipitation. The oxidation-reduction potential of groundwater is a key parameter for the iron precipitation, and eventually in maintaining a heat pump.

Figure 13 shows six areas where geothermal heat pump can be used in the study area. Area 1 is recommended for an open type Geo-HP. Iron scale may cause a problem in this area. An open type Geo-HP can be used in area 2 without any scaling problem. An open type Geo-HP can also be used in area 3, but the water flow has to be monitored because the flow may change with time (Fig. 10b). In the area 4 where most groundwaters are originated from meteoric precipitation, therefore the water level fluctuates depending on precipitation amount. A closed type Geo-HP system can be used without any problem. In the areas 5 and 6, a closed type Geo-HP is recommended, because calcite scaling and corrosion by high $\mathrm{Cl}$ concentration are expected if the open type Geo-HP is used.

\section{Conclusions}

Groundwater samples at the Sho river fan, Toyama Prefecture, Japan were geochemically examined to assess the applicability of groundwater to heat usage for air conditioning and melting of road snow. The shallow groundwaters are of meteoric origin and of a $\mathrm{Ca}-\mathrm{HCO}_{3}$ type. High geothermal gradient (twice as much as the other areas in Japan) found in the observation wells suggests existence of high geothermal sources under this region. The Sho river fan can be classified into 6 areas where either an open type or closed Geo-HP is better used, depending on the quality of groundwater from each area. The shallow groundwaters in most part of the study area are appropriate for heat usage by an open-type heat pump system. In some groundwater with high ORP, iron precipitation in the heat exchange is possible and Fe concentration should be monitored for the open type GeoHP. A closed type Geo-HP is recommended for groundwater which is oversaturated with iron and calcite precipitations, because of possible scaling by the precipitates in the pipes used in the closed type system.

Acknowledgments - The authors express their thanks to the late Professor H. Satake. The authors wish to thank to members of Naka Energy Institute, Mitsubishi Materials Corp., especially to $\mathrm{S}$. Ueta for helping us to analyze Fe concentration in groundwater samples. We like to thank M. Kusakabe and the anonymous reviewers for their critical and constructive comments which improved the earlier version of the manuscript.

\section{REFERENCES}

Andritsos, N. and Karabelas, A. J. (2003) Calcium carbonate scaling in a plate heat exchanger in the presence of particles. J. Heat Mass Transfer 46, 4613-4627.

Appelo, C. A. J. and Postma, D. (2005) Geochemistry, Groundwater and Pollution. 2nd ed., AA Balkema, Amsterdam, 649 pp.

Charette, M. A. and Sholkovitz, E. R. (2002) Oxidative precipitation of groundwater-derived ferrous iron in the subterranean estuary of a coastal bay. Geophys. Res. Lett. 29, 1-4.

Chen, C., Wang, C., Hsu, Y., Yu, S. and Kuo, L. (2010) Correlation between groundwater level and altitude variations in land subsidence area of the Choshuichi Alluvial Fan, Taiwan. Eng. Geol. 115, 122-131.

Chkirbene, A., Tsujimura, M., Charef, A. and Tanaka, T. (2009) Hydro-geochemical evolution of groundwater in an alluvial aquifer: Case of Kurokawa aquifer, Tochigi prefecture, Japan. Desalination 246, 485-495.

Craig, H. (1961) Standard for reporting concentrations of deuterium and oxygen-18 in natural waters. Science 133, 1833.

Dansgaard, W. (1964) Stable isotopes in precipitation. Tellus 16, 436-438.

Diao, N., Li, Q. and Fang, Z. (2004) Heat transfer in ground heat exchangers with groundwater advection. J. Thermal Sci. 43, 1203-1211.

Freedman, V. L., Waichler, S. R., Mackley, R. D. and Horner, J. A. (2012) Assessing the thermal environmental impacts of an groundwater heat pump in southeastern Washington State. Geothermics 42, 65-77.

Fujii, H., Inatomi, T., Itoi, R. and Uchida, Y. (2007) Development of suitability maps for ground-coupled heat pump systems using groundwater and heat transport models. Geothermics 36, 459-472.

Hidalgo, M. C. and Cruz-Sanjulian, C. (2001) Groundwater composition, hydrochemical evolution and mass transfer in a regional detrital aquifer (Baza basin, southern Spain). Appl. Geochem. 16, 745-758.

Huttrer, G. W. (1997) Geothermal heat pumps: An increasingly successful technology. Renew. Energ. 10, 481-488. 
Kato, S., Mizutani, Y., Uchida, T. and Iida, C. (1984) Geochemical study of ground water systems in the Shogawa Fan, Toyama. Chikyu-kagaku 18, 29-35 (in Japanese with English abstract).

Kushita, K., Katsumata, S., Shimizu, R., Kawada, S. and Ueda, A. (2012) Continuous evaluation test of an open type Geo$\mathrm{HP}$ at Nanto City, Toyama. Abst. Annual Meeting of Japan Geothermal Society, P27 (in Japanese).

Lee, J. and Hahn, J. (2006) Characterization of groundwater temperature obtained from the Korean national groundwater monitoring stations: Implications for heat pumps. J. Hydrol. 329, 514-526.

Lei, C., Peng, Z., Day, T., Yan, X., Bai, X. and Yuan, C. (2011) Experimental observation of surface morphology effect on crystallization fouling in plate heat exchangers. Inter. Commun. Heat Mass Trans. 38, 25-30.

Li, F., Pan, G., Tang, C., Zhang, Q. and Yu, J. (2008) Recharge source and hydrogeochemical evolution of shallow groundwater in a complex alluvial fan system, southwest of North China Plain. Environ. Geol. 55, 1109-1122.

Mizutani, Y. and Oda, M. (1983) Stable isotope study of ground water recharge and movement in the Shogawa Fan, Toyama. Chikyu-kagaku 17, 1-9 (in Japanese with English abstract).

Mizutani, Y., Satake, H. and Takashima, H. (1987) Tritium age of groundwaters from the Shogawa fan, Toyama. Chikyukagaku 21, 49-54 (in Japanese with English abstract).

Mizutani, Y., Satake, H., Yamabe, A., Miyachi, H., Mase, N. and Yamamura, K. (2001) Hydorogen and oxygen isotope ratios of groundwaters in shallow aquifers beneath the alluvial fan. J. Groundwater Hydrol. 43, 3-11 (in Japanese with English abstract).

Miyamoto, N. (2000) Stable isotopic compositions of groundwaters in Takaoka area. Bachelor thesis of University of Toyama, 35 pp. (in Japanese).

Ni, L., Li, H., Jiang, Y., Yao, Y. and Ma, Z. (2011) A model of groundwater seepage and heat transfer for single-well ground source heat pump systems. Appl. Therm. Eng. 31, 2622-2630.

Parkhurst, D. L. and Appelo, C. A. J. (1999) Users Guide to PHREEQC (Version2)-A Computer Program for Speciation, Batch-Reaction, One-Dimensional Transport, and Inverse Geochemical Calculations. Water-Resources Investigations Report, U.S. Department of the Interior, U.S. Geological Survey, 99-4259.

Russo, S. L., Taddia, G., Baccino, G. and Verda, V. (2011) Different design scenarios related to an open loop groundwater heat pump in a large building: Impact on subsurface and primary energy consumption. Energ. Buildings 43, 347-357.

Satake, H., Mukai, T. and Mizutani, Y. (1984) Environmental isotope hydrology of precipitation and river waters in the Hokuriku district, Japan. Annual Report of Hydrogen Iso- tope Research Center, University of Toyama 3, 45-56 (in Japanese with English abstract).

Schwertmann, U. and Murad, E. (1983) Effect of $\mathrm{pH}$ on the formation of goethite and hematite from ferrihydrite. Clays Clay Miner. 31, 277-284.

Sumi, Y., Nozawa, T. and Inoue, M. (1989) Geological map and instructions 10-21 (Isurugi - 1:50,000). Geological Survey of Japan, 45 pp.

Takahashi, N., Enomoto, M. and Satake, H. (2003) Measurement of water flow rate by deuterium dilution method. Report of Forum Saitama, 1-2 (in Japanees).

Takaoka, N. and Mizutani, Y. (1987) Tritiogenic ${ }^{3} \mathrm{He}$ in groundwater in Takaoka. Earth Planet. Sci. Lett. 85, 74-78.

Tomiyama, S., II, H., Miyaike, S., Hattori, R. and Ito, Y. (2009) Estimation of the sources and flow system of groundwater in Fuji-Gotenba area by stable isotopic analysis and groundwater flow simulation. Bunseki Kagaku 58, 865-872 (in Japanese with English abstract)

Tomiyama, S., II, H., Uehara, T. and Wakita, T. (2010) Stable isotopic analysis and groundwater flow simulation as potential tools for estimating groundwater sources used for food materials. Nippon Shokuhin Kagaku Kogaku Kaishi 57, 32-39 (in Japanese with English abstract).

Tomiyama, S., Ueda, A., Kitai, A. and Aoi, S. (2011) Estimation of the sources of groundwater used for food materials in Sayama city, Saitama prefecture, by geochemical methods and numerical simulation. Bunseki Kagaku 60, 131142 (in Japanese with English abstract).

Toyama Prefecture (ed.) (2006) Groundwater guidelines Toyama. Report of Toyama Prefecture, 29 pp. (in Japanese), available at http://www.pref.toyama.jp/cms_pfile/ 00001661/00066924.pdf

Tsuchihara, T., Yoshimoto, S., Ishida, S. and Imaizumi, M. (2011) Classification of recharge sources of groundwater in a Paddy Dominant Alluvial Fan Based on Geochemical and isotopic analyses. Bull. Nat. Inst. Rural Eng. 211, 2134 (in Japanese with English abstract).

Vanderzalm, J. L., Jeuken, B. M., Wischusen, J. D. H., Pavelic, P., Le Gal La Salle, C., Knapton, A. and Dillon, P. J. (2011) Recharge sources and hydrogeochemical evolution of groundwater in alluvial basins in arid central Australia. $J$. Hydrol. 397, 71-82.

Wang, Y., Wong, K. L., Liu, Q., Jin, Y. and Tu, J. (2012) Improvement of energy efficiency for an open-loop surface water source heat pump system via optimal design of water-intake. Energ. Buildings 51, 93-100.

Yuan, R., Song, X., Zhang, Y., Han, D., Wang, S. and Tang, C. (2011) Using major ions and stable isotopes to characterize recharge regime of a fault-influenced aquifer in Beiyishui River Watershed, North China Plain. J. Hydrol. 405, 512521. 